\title{
Hard X-ray variability of V404 Cygni during the 2015 outburst $^{\star}$
}

\author{
C. Sánchez-Fernández ${ }^{1}$, J. J. E. Kajava ${ }^{1,2,3}$, S. E. Motta ${ }^{4}$, and E. Kuulkers ${ }^{1,5}$
}

\author{
1 European Space Astronomy Centre (ESA/ESAC), Science Operations Department, 28691 Villanueva de la Cañada, Madrid, Spain \\ e-mail: Celia.Sanchez@sciops.esa.int \\ ${ }^{2}$ Finnish Centre for Astronomy with ESO (FINCA), University of Turku, Väisäläntie 20, 21500 Piikkiö, Finland \\ 3 Tuorla Observatory, Department of Physics and Astronomy, University of Turku, Väisäläntie 20, 21500 Piikkiö, Finland \\ ${ }^{4}$ University of Oxford, Department of Physics, Astrophysics, Denys Wilkinson Building, Keble Road, Oxford OX1 3RH, UK \\ 5 ESA/ESTEC, Keplerlaan 1, 2201 AZ Noordwijk, The Netherlands
}

Received 30 August 2016 / Accepted 22 March 2017

\begin{abstract}
Aims. Hard X-ray spectra of black hole binaries (BHB) are produced by Comptonization of soft seed photons by hot electrons near the black hole. The slope of the resulting energy spectra is governed by two main parameters: the electron temperature $\left(T_{\mathrm{e}}\right)$ and optical depth $(\tau)$ of the emitting plasma. Given the extreme brightness of V404 Cyg during the 2015 outburst, we aim to constrain the source spectral properties using an unprecedented time resolution in hard X-rays, and to monitor the evolution of $T_{\mathrm{e}}$ and $\tau$ over the outburst. Methods. We have extracted and analysed 602 X-ray spectra of V404 Cyg obtained by the IBIS/ISGRI instrument on-board INTEGRAL during the 2015 June outburst, using effective integration times ranging between 8 and $176000 \mathrm{~s}$. We fitted the resulting spectra in the 20-200 keV energy range.

Results. We find that while the light curve and soft X-ray spectra of V404 Cyg are remarkably different from those of other BHBs, the spectral evolution of V404 Cyg in hard X-rays and the relations between the spectral parameters are consistent with those observed in other BHBs. We identify a hard branch in which the $T_{\mathrm{e}}$ is anti-correlated with the hard X-ray flux, and a soft flaring branch in which the relation reverses. In addition, we find that during long X-ray plateaus detected at intermediate fluxes, the thermal Comptonization models fail to describe the spectra. However, the statistics improve if we allow $N_{\mathrm{H}}$ to vary freely in the fits to these spectra.

Conclusions. We conclude that the hard branch in V404 Cyg is analogous to the canonical hard state of BHBs. V404 Cyg never seems to enter the canonical soft state, although the soft flaring branch bears resemblance to the BHB intermediate state and ultraluminous state. The X-ray plateaus are likely the result of absorption by a Compton-thick outflow $\left(N_{\mathrm{H}} \gtrsim 10^{24} \mathrm{~cm}^{-2}\right)$ which reduces the observed flux by a factor of about 10 . Variable covering of the central source by this Compton-thick material may be the reason for the complicated light curve variability, rather than intrinsic source variability.
\end{abstract}

Key words. accretion, accretion disks - black hole physics - X-rays: binaries - X-rays: individuals: V404 Cyg

\section{Introduction}

Black hole (BH) binary systems (BHBs) can go through various spectral states that are thought to be caused by changes in the accretion geometry and accretion rates close to the $\mathrm{BH}$, although the actual details still remain debated (Remillard \& McClintock 2006; Belloni \& Motta 2016). The two most common states are the hard and the soft states (see, e.g., Done et al. 2007; Poutanen \& Veledina 2014, for review). In the hard state, the spectrum can be described by a power law with a variable cut-off energy around $60-150 \mathrm{keV}$, which is thought to result from Comptonization of soft seed photons by a population of hot electrons located in an optically thin region close to the BH (Shapiro et al. 1976; Narayan \& Yi 1995). The high energy cut-off suggests a thermal distribution of electrons with temperatures in the range 30-100 keV (Sunyaev \& Truemper 1979; Gierliński et al. 1999). Occasionally, a hard excess has been observed above $100 \mathrm{keV}$ which suggests the presence of non-thermal electrons as well (see e.g. McConnell et al. 2002; Wardziński et al. 2002; Joinet et al. 2007; Droulans et al. 2010) either in the corona/hot flow or in the base of the jet (e.g.,

\footnotetext{
* Based on observations with INTEGRAL, an ESA project with instruments and science data centre funded by ESA member states (especially the PI countries: Denmark, France, Germany, Italy, Switzerland, Spain) and with the participation of Russia and the USA.
}

Zdziarski et al. 2012). In the soft state, thermal emission peaking at $\sim 1 \mathrm{keV}$, from a cool, optically thick, geometrically thin accretion disk dominates the spectrum (Shakura \& Sunyaev 1973; Esin et al. 1997). A weak, hard X-ray tail extending up to the $\mathrm{MeV}$ range is also detected (Zdziarski et al. 2017). This tail is thought to originate from Comptonized emission by nonthermal electrons in discrete flares on top of the accretion disk (McConnell et al. 2002). During transitions between the hard and soft states, BHBs pass through additional intermediate states, which show characteristic features of both (see e.g. Ebisawa et al. 1994; Malzac et al. 2006; Belloni \& Motta 2016). On rare occasions, some systems may pass also through the so-called ultra-luminous state (Motta et al. 2012), which is also called the very high state or anomalous state; this is an intermediate state characterized by both a strong thermal component and a very strong and steep hard X-ray tail (Done et al. 2007).

One of the current observational challenges in this context is to determine the electron temperature, $T_{\mathrm{e}}$, and optical depth, $\tau$, of the Comptonizing medium. These together determine the spectral slope of the Comptonized spectrum (e.g. Beloborodov 1999). Because the cut-off energies are found around $100 \mathrm{keV}$, where usually the instrumental response is low, observations sensitive enough to constrain these parameters have only been available for a few sources and typically require long exposures. Observations in the hard state of GRO J0422+32 (Esin et al. 1998), 
GX 339-4 (Wardziński et al. 2002; Motta et al. 2009), XTE J1550-564 (Rodriguez et al. 2003), Cyg X-1 (Del Santo et al. 2013) and Swift J1753.5-0127 (Kajava et al. 2016) show an anti-correlation between the electron temperature, $T_{\mathrm{e}}$ (or high energy cut-off) and the X-ray flux, accompanied by a correlation between the plasma optical depth, $\tau$, and the X-ray flux (Wardziński et al. 2002). These relations reverse during the hard to soft state transitions. Observations of these transitions in Cyg X-1 (Phlips et al. 1996; Del Santo et al. 2013), GRO J171924 (Esin et al. 1998), GRO J1655-40 (Joinet et al. 2008) and GX 339-4 (Motta et al. 2009) show an increasing $T_{\mathrm{e}}$ with increasing flux, while the optical depth $\tau$ decreases (Joinet et al. 2008; Del Santo et al. 2013). The cut-off is significantly present during the hard and intermediate states, and it disappears when the source reaches the soft state.

The extremely bright outburst of V404 Cyg in June 2015 provides a unique data set to perform high time-resolved spectroscopy in high energies and study in detail the evolution of the parameters describing the Comptonizing plasma. We present here the results of spectral analysis of IBIS/ISGRI data in the 20$200 \mathrm{keV}$ energy range over the period 18-28 June 2015, when the source was brightest.

V404 Cyg is a transient low-mass X-ray binary (LMXB) consisting of a $9.0_{-0.6}^{+0.2} M_{\odot} \mathrm{BH}$ accreting mass from a K3 III companion (Khargharia et al. 2010) in a $6.5 \mathrm{~d}$ orbit (Casares et al. 1992). It is located at a distance $d=2.39 \pm 0.14 \mathrm{kpc}$ (Miller-Jones et al. 2009). V404 Cyg was first detected in optical wavelengths during two outbursts in 1938 and 1956 (Richter 1989) and later in X-rays during a third outburst in 1989 (Makino 1989; Marsden 1989). The 1989 outburst was characterized by extreme flaring activity, several flux levels above the Crab (Tanaka 1989; Oosterbroek et al. 1996). After $\sim 26 \mathrm{yr}$ in quiescence, the onset of a new outburst was detected by Swift/BAT, MAXI and Fermi/GBM on 15 June 2015 (Barthelmy \& Sbarufatti 2015; Negoro et al. 2015; Younes 2015). This outburst, which triggered the most intensive multiwavelength observing campaign performed so far on a transient BHB, lasted until early August 2015 (Sivakoff et al. 2015). During the first 10 days, the source exhibited violent flaring activity on timescales of subseconds to hours in all wavelengths: $\gamma$-rays (Loh et al. 2016) X-rays (Rodriguez et al. 2015; Roques et al. 2015; Jenke et al. 2016; Walton et al. 2016), optical (Gandhi et al. 2016; Kimura et al. 2016; Muñoz-Darias et al. 2016), infrared (Eikenberry et al. 2016) and millimeter/submillimeter and radio (Tetarenko et al. 2015). In some major flares, V404 Cyg reached fluxes around 50 and $40 \mathrm{Crab}$ in soft and hard X-rays, respectively (Segreto et al. 2015; Rodriguez et al. 2015). The peak of the outburst was reached on 26 June, and the flux dropped immediately afterwards (Ferrigno et al. 2015; Walton et al. 2015) slowly fading to quiescence over the subsequent weeks (Sivakoff et al. 2015).

\section{Observations and data analysis}

V404 Cyg was observed by INTEGRAL, the INTErnational Gamma-Ray Astrophysics Laboratory (Winkler et al. 2003) in a series of Target of Opportunity observations scheduled between 17 June 2015 and 13 July 2015 (MJD 57 556-57 582; revolutions 1554-1563; Kuulkers 2015). We present here the analysis of the available IBIS/ISGRI data (Lebrun et al. 2003), obtained during revolutions 1554-1558 (18-28 June 2015; MJD 57 19151201 ), which cover the epoch of intense flaring activity and the beginning of the outburst decay. These observations provide data sensitive enough to study in detail the properties of the Comptonizing medium.

\subsection{Data reduction}

The IBIS/ISGRI data reduction was performed with the Offline Scientific Analysis software (OSA; Courvoisier et al. 2003) $\mathrm{v} 10.2$, using the latest calibration files. The data were processed following standard IBIS/ISGRI reduction procedures.

The spectral extraction was performed using good time interval files (GTIs) of variable duration, which are defined to provide source spectra of comparable $\mathrm{S} / \mathrm{N}$ regardless of the source flux. The GTI selection was based on the source light curves distributed by the INTEGRAL Science Data Center (ISDC; Kuulkers 2015). The GTIs were defined sequentially, and their start/end times were selected such that during each time interval $4 \times 10^{5}$ counts were accumulated in the IBIS/ISGRI $25-60 \mathrm{keV}$ band. This GTI selection was found to be an optimal compromise between time resolution and the ability to constrain the $T_{\mathrm{e}}$ values, particularly for the softer spectra in the sample. Using this strategy, we extracted 602 spectra, with effective exposure times in the range 8 to $176000 \mathrm{~s}$. We binned the IBIS/ISGRI response matrix in the energy range $20-500 \mathrm{keV}$ using 28 channels of variable logarithmic widths. We restricted the spectral fits to the $20-200 \mathrm{keV}$ energy range to remove potential background contamination, and the contribution of additional spectral components above $200 \mathrm{keV}$, such as hard non-thermal tails (Rodriguez et al. 2015; Roques et al. 2015) or hard X-ray emission caused by positron annihilation (Siegert et al. 2016). In our fits we ignored the energy bin around $50 \mathrm{keV}$ due to calibration uncertainties and added 3 per cent systematic errors to the spectral bins. The IBIS/ISGRI X-ray spectra were fit via XSPEC v12.8.2 (Arnaud 1996), adopting the $\chi^{2}$ statistics. Errors provided below are quoted at the $1 \sigma$ confidence level $\left(\Delta \chi^{2}=1\right.$ for one parameter of interest).

\subsection{Spectral modelling}

We show in Fig. 1 a sample of the IBIS/ISGRI spectra of V404 Cyg analysed in this work. On most occasions, the spectra have a shape that is power-law like, modified by a cut-off at high energies, which is consistent with a thermal Comptonization spectrum (see Fig. 1a). Therefore, we used Comptonization models to fit our data: NTHCOMP (Zdziarski et al. 1996; Życki et al. 1999) and CoMPPS (Poutanen \& Svensson 1996). These models provide a description of the continuum produced by thermal Compton up-scattering of soft X-ray photons. The NTHCOMP model is parameterized by a power-law index $\Gamma$ and an electron temperature $T_{\mathrm{e}}$. The coMPPS parameters are the electron temperature $T_{\mathrm{e}}$ and optical depth $\tau$. Although a thermal component was never detected during the June 2015 outburst (Motta et al., in prep.), in our fits we fixed the seed photon temperature $T_{\mathrm{bb}}=0.1 \mathrm{keV}$, as fits by Motta et al. (2017) to the source spectra over the 0.6-200 keV energy range, with Comptonization models are consistent with this value.

In some cases, a high-energy cut-off is either weakly significant or not statistically required by the data (see Fig. 1b). These spectra can still be fit using Comptonization models, but fixing $T_{\mathrm{e}}$ to an arbitrary high value $\left(T_{\mathrm{e}}=999 \mathrm{keV}\right)$. To account for both possibilities, we carried out two independent fitting runs per model. In the first fitting run, we fit every spectrum leaving $T_{\mathrm{e}}$ as a free parameter, while in the second fitting run we fixed it to $T_{\mathrm{e}}=999 \mathrm{keV}$. Then, the Bayesian information criterion (BIC; 

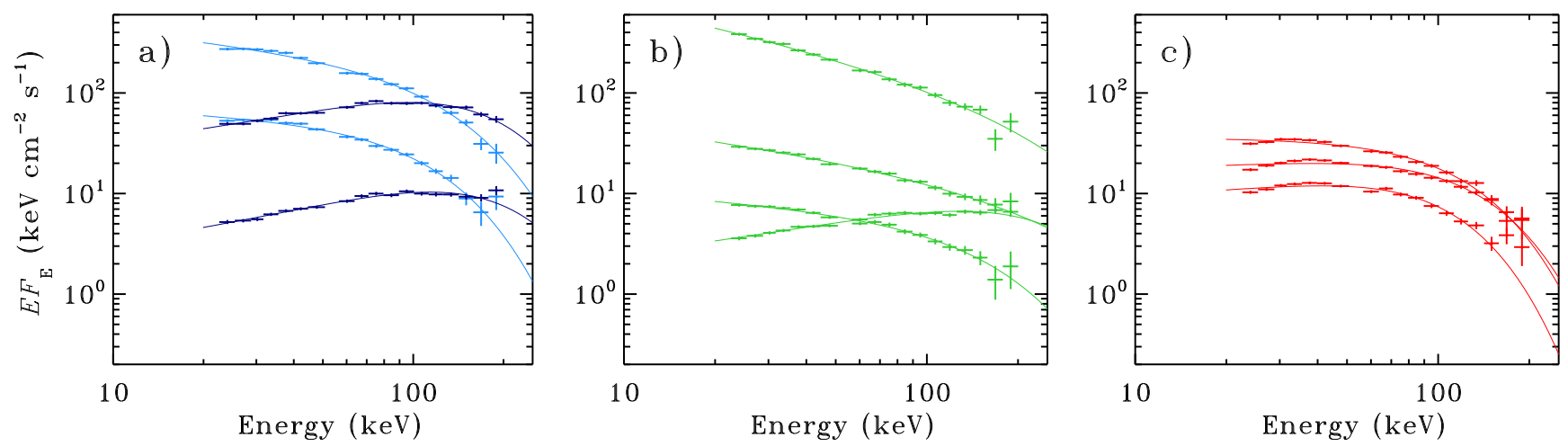

Fig. 1. Example of the spectra analysed in this work. Panel a) Comptonized spectra showing a cut-off at high energies within the IBIS/ISGRI energy range. We classify these spectra in two groups: hard ( $\Gamma \leq 1.7$; dark blue) and soft $(\Gamma>1.7$; light blue). The hardness selection is based on the $\Gamma$ values derived using the NTHCOMP model. From top to bottom, the effective integration times for these spectra are $36,170 \mathrm{~s}$ ( $\Gamma>1.7 \mathrm{spectra})$ and $91,1285 \mathrm{~s}(\Gamma \leq 1.7 \mathrm{spectra})$. Panel b) Comptonized spectra for which $T_{\mathrm{e}}$ cannot be constrained by our data. We fixed the electron temperature to the value $T_{\mathrm{e}}=999 \mathrm{keV}$ in these spectral fits. We note the range in fluxes and hardness presented by these spectra. The effective integration times for these spectra are $8,403,1730$ and $2251 \mathrm{~s}$ (top to bottom). Panel c) spectra for which a Comptonized model was not statistically favoured by our model selection criteria $(p$-value $<0.05)$. These spectra are predominantly found during the X-ray plateaus detected at fluxes $F_{\mathrm{X}} \sim 5 \times 10^{-8} \mathrm{erg} \mathrm{cm}^{-2} \mathrm{~s}^{-1}$, as explained in the text. The effective integration times for these spectra are 297,579 and $912 \mathrm{~s}$ (top to bottom).

Schwarz 1978) was independently applied to the results obtained for every spectrum, to select the best fit to the data. We computed the BIC with the following approximation: $\mathrm{BIC}=\chi^{2}+k \ln (n)$, where $k$ is the number of parameters in the model, and $n$ is the number of channels in the spectral fits. In a model selection process, the optimal model is identified by the minimum value of BIC. A lower BIC implies either fewer explanatory variables, a better fit, or both. Kass (1995) set the strength of the evidence against the model with the higher BIC to be strong if $\Delta \mathrm{BIC}>6$, which we adopted as the limit for model selection. This approach was applied to the NTHCOMP and COMPPS fits. The results of this analysis are described in Sects. 3.1-3.3, and shown in Figs. 2A.3. In these figures the data are presented according to the following colour convention:

- Blue points are used to highlight those fits where the $\triangle \mathrm{BIC}$ model selection favoured a Comptonization model with a constrained electron temperature $T_{\mathrm{e}}$, further divided in two groups: hard spectra $(\Gamma \leq 1.7$; dark blue) and soft spectra $(\Gamma>1.7$; light blue). Some of these spectra are shown in Fig. 1a. The latter classification is based on the $\Gamma$ values derived from the NTHCOMP fits, and then applied to the COMPPS fits.

- Green points correspond to those spectra where $T_{\mathrm{e}}$ could not be constrained by our fits (i.e. $T_{\mathrm{e}}$ fixed at $999 \mathrm{keV}$ ). Some of these spectra are shown in Fig. 1b.

- Additionally, we computed the corresponding $p$-value of the fit with respect to the data for every fit. We mark the spectra where $p<0.05$ with red symbols. Some of these spectra are shown in Fig. 1c.

To improve the fits to the latter group of spectra, we also explored the possibility that these were affected by heavy absorption. The results of these additional fits are presented in Sect. 3.4.

\section{Results}

\subsection{Parameter evolution}

We present in Fig. 2 the time evolution of the source flux computed in the 20-200 keV energy range, together with the evolution of the spectral parameters derived using NTHCOMP $\left(\Gamma, T_{\mathrm{e}}\right)$ and COMPPS $\left(\tau, T_{\mathrm{e}}\right)$ and the corresponding $\chi_{v}^{2}$ values.
Hereafter we refer to the flux in the $20-200 \mathrm{keV}$ energy range as $F_{\mathrm{x}}$.

\subsubsection{EPOCH 1: flaring activity}

During the period MJD 57 191-57 193 (Rev. 1554; EPOCH 1 in Fig. 2a) intense flaring activity was detected on timescales of minutes to hours. The flares reached peak fluxes of $F_{\mathrm{x}} \sim 55 \times 10^{-8} \mathrm{erg} \mathrm{cm}^{-2} \mathrm{~s}^{-1}$, while between flares we measure fluxes below $F_{\mathrm{x}} \sim 2 \times 10^{-8} \mathrm{erg} \mathrm{cm}^{-2} \mathrm{~s}^{-1}$. Over this period, the source spectrum was hard $(\Gamma \leq 1.7)$ and only softened when $F_{\mathrm{x}}$ increased above $\gtrsim 25 \times 10^{-8} \mathrm{erg} \mathrm{cm}^{-2} \mathrm{~s}^{-1}$ (i.e. during the peaks of the flares). The value $T_{\mathrm{e}}$ is well constrained during the X-ray flares with values in the range $30-100 \mathrm{keV}$ (NTHCOMP) or 30$70 \mathrm{keV}$ (COMPPS). Between flares $T_{\mathrm{e}}$ cannot be constrained in our spectral fits and the X-ray spectrum is consistent with a hard power law $(\Gamma \leq 1.7)$ with no cut-off (see Fig. 1b). Similar results were obtained by Natalucci et al. (2015), who analysed this data set using a different time resolution, and Roques et al. (2015), who analysed contemporaneous INTEGRAL/SPI data.

In EPOCH $1 \tau$ varied between 2 and 5. It displayed higher values $(\tau \gtrsim 3.5)$ when the spectrum was hard $(\Gamma \leq 1.7)$ and decreased $(\tau \lesssim 3.5)$ as the spectrum softened $(\Gamma>1.7$; see Fig. $2 d)$.

\subsubsection{EPOCH 2: spectral softening, state transitions, and X-ray plateaus}

During the intervals MJD 57 193.5-57 195.5 and MJD 57 196.757197.4 (Rev. 1555-1556; EPOCH 2 in Fig. 2a) the flaring activity persisted. Peak fluxes of $F_{\mathrm{p}} \sim 60-80 \times 10^{-8} \mathrm{erg} \mathrm{cm}^{-2} \mathrm{~s}^{-1}$ were measured. Between flares, we find again fluxes $F_{\mathrm{x}} \lesssim$ $2 \times 10^{-8} \mathrm{erg} \mathrm{cm}^{-2} \mathrm{~s}^{-1}$. In general, in EPOCH 2 V404 Cyg displayed softer spectra than during EPOCH 1 even at the lowest count rates, when spectra with $\Gamma \sim 2.3$ without a cut-off were frequently observed. Only one of the X-ray flares (detected around MJD 57 195.15) displayed a hard X-ray spectrum $(\Gamma \leq 1.7)$. Several transitions between Comptonized hard $(\Gamma \leq 1.7)$ and soft $(\Gamma \sim 3)$ spectra with unconstrained $T_{\mathrm{e}}$ occurred.

In EPOCH 2, there is more scatter in the measured $T_{\mathrm{e}}, \tau$ and $\Gamma$ parameters than in EPOCH 1. Also, the relation between $F_{\mathrm{x}}$ and $\Gamma$ is complex: while during some flares $\Gamma$ was roughly 
A\&A 602, A40 (2017)

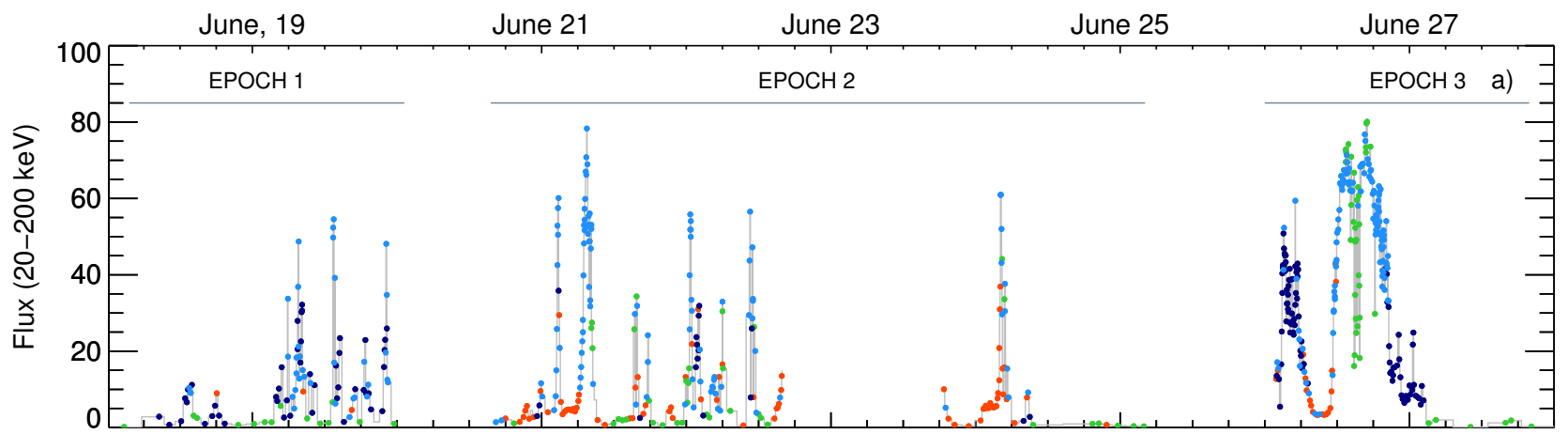

nthcomp

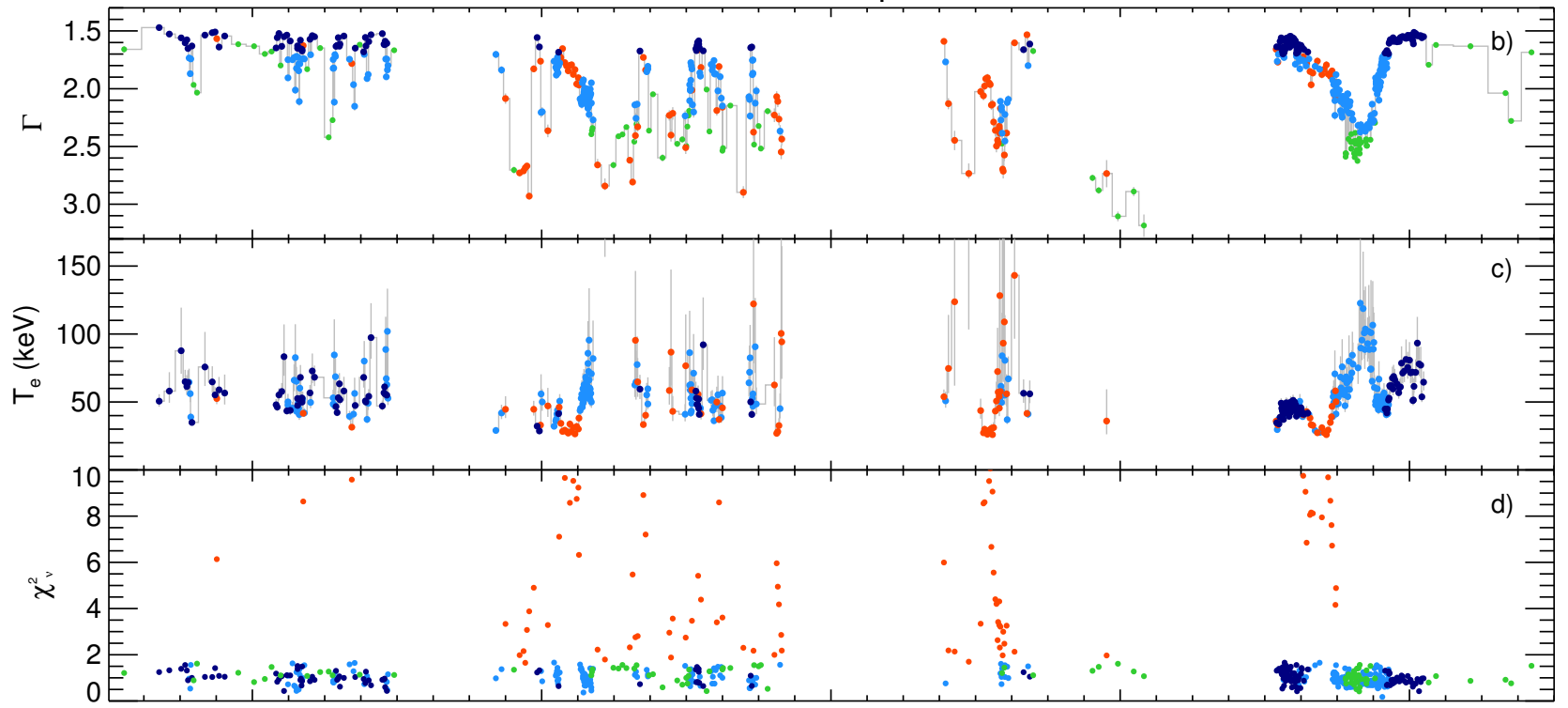

compPS

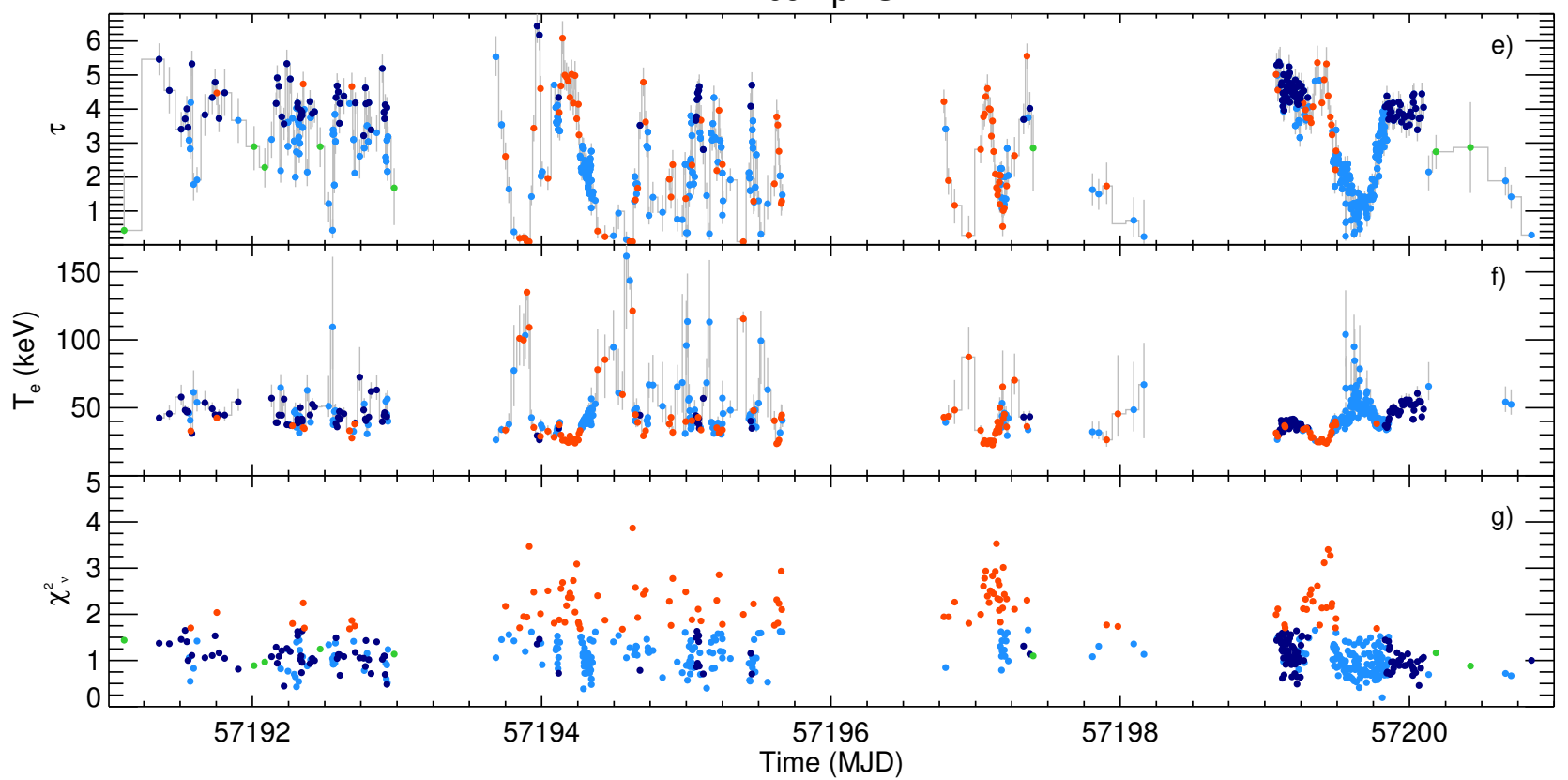

Fig. 2. Evolution of the flux and spectral parameters of V404 Cyg during the June 2015 flaring episodes. Panel a) source flux (20-200 keV) in units of $10^{-8} \mathrm{erg} \mathrm{cm}^{-2} \mathrm{~s}^{-1}$. Panels b), c), d) NTHCOMP fitting parameters (power-law index, $\Gamma$, electron temperature, $T_{\mathrm{e}}$ and $\chi_{v}^{2}$ ). Panels e), f), g) COMPPS fitting parameters (optical depth, $\tau$, electron temperature, $T_{\mathrm{e}}$ and $\chi_{v}^{2}$ ). Green, blue and red symbols are used to highlight the best-fitting model, according to our model selection criteria (Sect. 2.2). Blue: comptonization models with constrained $T_{\mathrm{e}}$ (Fig. 1a) further divided into hard ( $\Gamma \leq 1.7$; dark blue) and soft spectra ( $\Gamma>1.7$; light blue). Green: comptonization models with unconstrained $T_{\mathrm{e}}$ (Fig. 1b). Red: comptonization models with $p$-value $<0.05$ fits. (Fig. $1 \mathrm{c}$ ); $\chi_{v}^{2} \gtrsim 2$ values are only obtained during the X-ray plateaus observed at $F_{\mathrm{x}} \sim 5 \times 10^{-8} \mathrm{erg} \mathrm{cm}^{-2} \mathrm{~s}^{-1}$. 
constant (e.g. flare on MJD 57 194.10, Fig. A.1), in other flares the spectrum hardened (e.g. flare on MJD 57 195.5) or softened during the entire flare (e.g. flare on MJD 57 194.3, Fig. A.1). During the flares, $T_{\mathrm{e}}$ displayed values in the range $30-120 \mathrm{keV}$ (NTHCOMP) or 20-150 keV (COMPPS). Very soft spectra $(\Gamma \sim 3)$ with unconstrained $T_{\mathrm{e}}$ were detected by the end of EPOCH 2, when the flux dropped below $\sim 2 \times 10^{-8} \mathrm{erg} \mathrm{cm}^{-2} \mathrm{~s}^{-1}$.

One additional feature over this period is the detection of $\mathrm{X}$-ray plateaus (i.e. non-varying flux periods) at intermediate fluxes $\left(F_{\mathrm{x}} \sim 5 \times 10^{-8} \mathrm{erg} \mathrm{cm}^{-2} \mathrm{~s}^{-1}\right)$. These plateaus last several hours and happen in between successive $\mathrm{X}$-ray flares (red points in Fig. 2; see also Rodriguez et al. 2015). For a closer view of one of these plateaus see Fig. A.1. We consistently obtain $p$-values $<0.05$ and $\chi_{v}^{2}>1.8$ when modelling the plateau spectra using Comptonization models (NTHCOMP or COMPPS), which also provide systematically lower electron temperatures for these data points $\left(T_{\mathrm{e}} \sim 30 \mathrm{keV}\right)$ than those derived in fits to contemporaneous flare spectra $\left(T_{\mathrm{e}} \sim 50 \mathrm{keV}\right)$. Joint spectral fits to simultaneous Swift/XRT, INTEGRAL/JEMX and INTEGRAL/ISGRI data by Motta et al. (2017) obtained during the plateau observed on MJD 57194 , showed a high absorption $\left(N_{\mathrm{H}} \approx 1.4 \times 10^{24} \mathrm{~cm}^{-2}\right)$ over a dominant reflection component.

Adding an absorption component (TBABS; $N_{\mathrm{H}} \gtrsim 10^{24} \mathrm{~cm}^{-2}$ ) to the fits to the plateau spectra (as described in detail in Sect. 3.4), we obtain $T_{\mathrm{e}} \sim 50 \mathrm{keV}$, in better agreement with the values derived during contemporaneous flares.

\subsubsection{EPOCH 3: major flares and onset of outburst decay}

Between MJD 57199.05 and MJD 57200.10 we observed two major flares separated by a long X-ray plateau, similar to those seen in EPOCH 2. The first flare (MJD 57 199.05-57 199.15; Fig. A.2) reached a peak flux $F_{\mathrm{x}} \sim 55 \times 10^{-8} \mathrm{erg} \mathrm{cm}^{-2} \mathrm{~s}^{-1}$. During this flare, the spectrum was hard $(\Gamma \leq 1.7)$, contrary to the softer flares detected in EPOCH 2 . We measure roughly constant electron temperatures $\left(T_{\mathrm{e}} \sim 50 \mathrm{keV}\right)$ and an optical depth $\tau$ in the range [4-5.5], which decreased as the flare proceeded. During the subsequent X-ray plateau a flux $F_{\mathrm{x}} \sim 5 \times 10^{-8} \mathrm{erg} \mathrm{cm}^{-2} \mathrm{~s}^{-1}$ was measured (Fig. A.2). The plateau lasted $\sim 0.15$ day.

The plateau was followed by a major X-ray flare (MJD 57 199.50-57 199.80; Fig. A.3) during which the source reached the highest fluxes measured during the 2015 outburst $\left(F_{\mathrm{x}} \sim 80 \times 10^{-8} \mathrm{erg} \mathrm{cm}^{-2} \mathrm{~s}^{-1}\right)$. The flare had two peaks separated by a $\sim 1 \mathrm{~h}$ drop in flux (from $\sim 70$ to $\sim 20 \times 10^{-8} \mathrm{erg} \mathrm{cm}^{-2} \mathrm{~s}^{-1}$ and back to $\left.\sim 80 \times 10^{-8} \mathrm{erg} \mathrm{cm}^{-2} \mathrm{~s}^{-1}\right)$. Over the flare rise and decay we find a Comptonized, soft, X-ray spectrum that softened as $F_{\mathrm{x}}$ increased and hardened as $F_{\mathrm{x}}$ decreased. The value $T_{\mathrm{e}}$ also evolved in correlation with the flux variations and reached values above $\sim 130 \mathrm{keV}$ (NTHCOMP) or $\sim 90 \mathrm{keV}$ (COMPPS) during the peak of the flare. On some occasions around the peak of the flare $T_{\mathrm{e}}$ is not constrained by our fits (NTHCOMP).

During the flare decay we find an abrupt drop in flux (from $\sim 45$ to $\sim 15 \times 10^{-8} \mathrm{erg} \mathrm{cm}^{-2} \mathrm{~s}^{-1}$ ), which happened in less than half an hour. The drop in flux was accompanied by a transition to harder spectra, characterized by a roughly constant power-law index $(\Gamma \gtrsim 1.5)$, increasing $T_{\mathrm{e}}$ and decreasing $\tau$. After the transition, the flux decay continued at a roughly constant $\Gamma$. As the flux evolved towards quiescence values, the spectrum softened again, $T_{\mathrm{e}}$ was unconstrained and the optical depth, $\tau$, decreased. The two lowest flux spectra in Fig. 1b correspond to this period.

\subsection{Parameter distributions}

The distributions of the spectral parameters derived in this analysis are shown in Fig. 3. The integration times of the spectra are flux dependent, and consequently the parameter distributions are skewed towards higher fluxes when the integration times are shorter and therefore the sampling is more frequent.

The $F_{\mathrm{x}}$ distribution is shown in Figs. $3 \mathrm{a}$ and d. We measure fluxes in the range [0.01-80] $\times 10^{-8} \mathrm{erg} \mathrm{cm}^{-2} \mathrm{~s}^{-1}$ with a peak in the distribution at $\sim 50 \times 10^{-8} \mathrm{erg} \mathrm{cm}^{-2} \mathrm{~s}^{-1}$. We do not find hard Comptonized spectra $(\Gamma \leq 1.7)$ when $F_{\mathrm{x}} \gtrsim 50 \times 10^{-8} \mathrm{erg} \mathrm{cm}^{-2} \mathrm{~s}^{-1}$. We find soft Comptonized spectra $(\Gamma>1.7)$ for fluxes in the range [4-80] $\left(\times 10^{-8} \mathrm{erg} \mathrm{cm}^{-2} \mathrm{~s}^{-1}\right)$. Comptonized spectra with unconstrained electron temperatures are predominantly found at the lowest and highest fluxes. Spectra not compatible with Comptonized models are predominant in the range of fluxes [1.5-6] $\times 10^{-8} \mathrm{erg} \mathrm{cm}^{-2} \mathrm{~s}^{-1}$, with a peak in the distribution at $5 \times 10^{-8} \mathrm{erg} \mathrm{cm}^{-2} \mathrm{~s}^{-1}$.

The photon index ( $\Gamma$; Fig. $3 b$ ) was derived using the NTHcoMP model. The distribution of $\Gamma$ values is asymmetric with a peak at $\Gamma \sim 1.7$ and a tail extending to $\Gamma \sim 3.0$. We measure $\Gamma$ values in the range [1.5-2.4] for the Comptonized spectra with constrained $T_{\mathrm{e}}$. All the Comptonized spectra softer than $\Gamma \gtrsim 2.4$ display unconstrained $T_{\mathrm{e}}$ in our NTHCOMP fits. There are also a fraction of hard spectra $(\Gamma \sim 1.7)$ with unconstrained $T_{\mathrm{e}}$. The spectra detected during X-ray plateaus display $\Gamma$ values in the range [1.6-3.0] with a peak in the distribution at $\Gamma=1.8$.

The optical depth of the Comptonizing plasma ( $\tau$; Fig. 3e), which is derived via COMPPS shows a large scatter in values, in the range [0.1-5.0]. The hard and soft spectra have different $\tau$ distributions. For the hard spectra $(\Gamma \leq 1.7)$ we derive $\tau$ values in the range [3-5.5]. For the softer spectra $(1.7<\Gamma \leq 2.4)$, we derive $\tau$ values in the range [0.1-4.5].

The electron temperatures that we derive via the NTHCOMP and COMPPS models (Figs. 3c and f) display similar distributions with a narrow peak at moderate temperatures (NTHCOMP: $45 \mathrm{keV}$; COMPPS: $35 \mathrm{keV}$ ) and a tail extending up to $\sim 150 \mathrm{keV}$. However, the distribution of $T_{\mathrm{e}}$ derived using NTHCOMP is broader than the distribution of values obtained from the COMPPS fits. We find more spectra with unconstrained $T_{\mathrm{e}}$ using NTHCOMP than using COMPPS, which is probably because of the systematically lower $T_{\mathrm{e}}$ values derived using COMPPS. The electron temperatures derived for Comptonized hard $(\Gamma \leq 1.7)$ and soft spectra $(\Gamma>1.7)$ have consistent values, but we note that the tail of the $T_{\mathrm{e}}$ distribution extends to higher energies for the soft Comptonized spectra than for the hard spectra. The fits to the spectra obtained during X-ray plateaus provide a broad $T_{\mathrm{e}}$ distribution, which peaks at lower energies than the $T_{\mathrm{e}}$ distribution derived for the Comptonized spectra $(\sim 25 \mathrm{keV})$ and extend up to $\sim 130 \mathrm{keV}$.

\subsection{Parameter relations}

The flux dependencies of the photon index and optical depth are presented in the $F_{\mathrm{x}}-\Gamma$ and $F_{\mathrm{x}}-\tau$ diagrams (Figs. 4a, d). We find that the hard spectra in our sample $(\Gamma \leq 1.7)$ occupy a region in the $F_{\mathrm{x}}-\Gamma$ diagram that is reminiscent of the hard state in the BHB Hardness-Intensity Diagram (HID; Homan et al. 2001; Belloni 2004; Fender et al. 2004; Dunn et al. 2010). In this region, which we call hereafter the hard branch, the spectrum softens (from $\Gamma \approx 1.5$ to $\Gamma \approx 1.7$ ) while the flux increases and the optical depth increases with increasing flux from $\tau \sim 3$ to $\tau \sim 5.5$ (Fig. $4 \mathrm{~d}$ ). The hard spectra also occupy defined regions in the $F_{\mathrm{x}}-T_{\mathrm{e}}, \Gamma-T_{\mathrm{e}}$, and $\tau-T_{\mathrm{e}}$ diagrams (Figs. 4b, e, c, f). In these hard branch(es), 

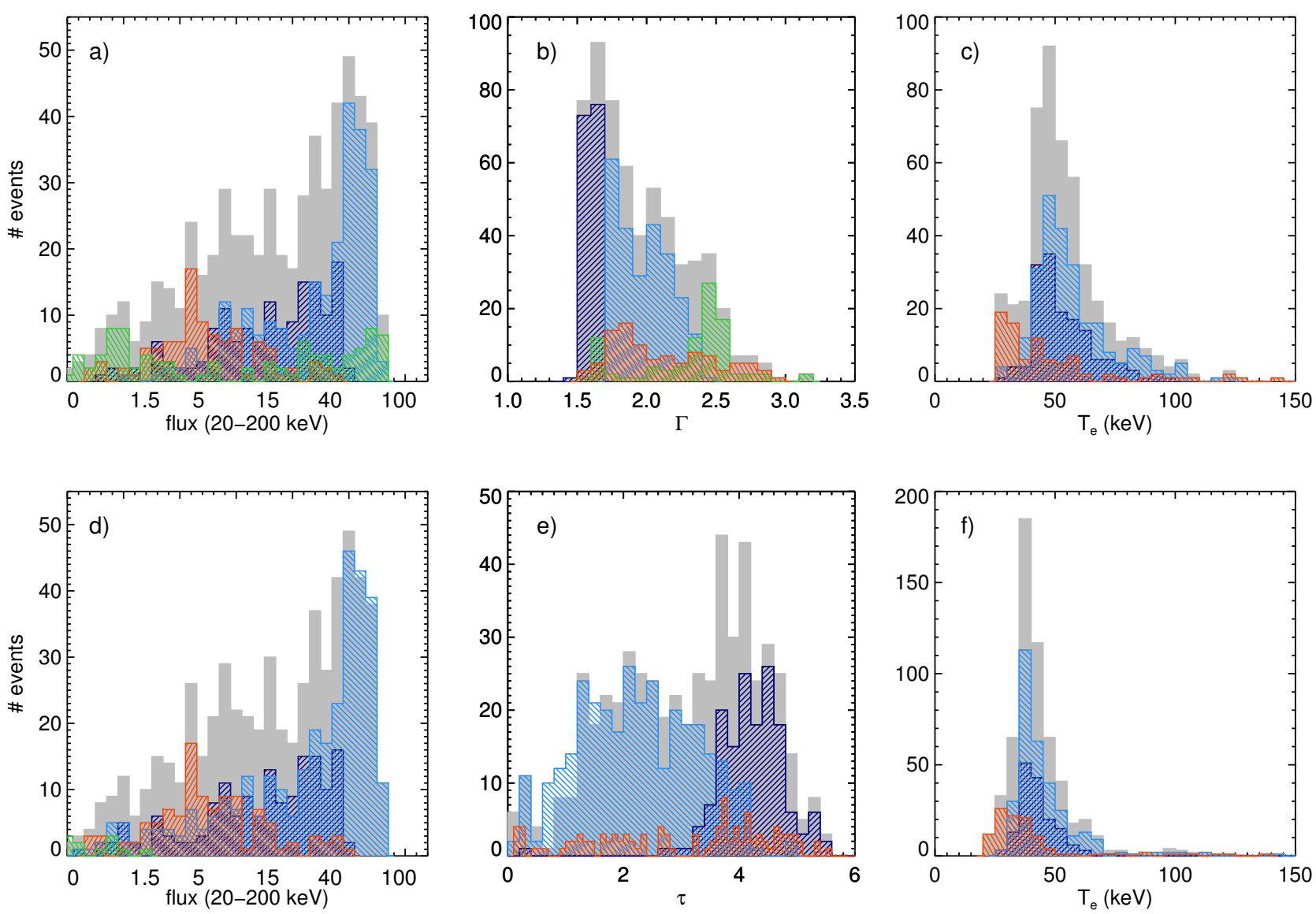

Fig. 3. Distribution of the spectral parameters obtained in the fits to the IBIS/ISGRI spectra of V404 Cyg analysed in this work. Grey bars are used to describe the total parameter distribution. Green, blue, and red symbols are used to highlight the best-fitting model, according to our model selection criteria (Sect. 2.2). Blue: comptonization models with constrained $T_{\mathrm{e}}$ (Fig. 1a) further divided into hard ( $\Gamma \leq 1.7$; dark blue) and soft spectra $\left(\Gamma>1.7\right.$; light blue). Green: comptonization models with unconstrained $T_{\mathrm{e}}$ (Fig. 1b). Red: $p$-value $<0.05$ fits. (Fig. $1 \mathrm{c}$ ). In the top panels we show the distribution of the NTHCOMP parameters, while in the bottom panels we show the COMPPS parameters.

$T_{\mathrm{e}}$ is anti-correlated with $F_{\mathrm{x}}$ (Fig. $\left.4 \mathrm{~b}, \mathrm{e}\right)$. We also find that $T_{\mathrm{e}}$ is anti-correlated with $\Gamma$ and $\tau(4 \mathrm{c}, \mathrm{f})$.

The soft $(\Gamma>1.7)$ spectra in our sample, detected during the brightest X-ray flares, occupy a distinct region in the $F_{\mathrm{x}}-\Gamma$ diagram (Fig. 4a) which we call hereafter the soft flaring branch. In the soft flaring branch the spectrum still softens as the flux increases (Fig. 4a) even though most of the parameter dependencies are reversed with respect to the hard branch: $\tau$ is seen to decrease with increasing flux (Fig. 4d), $F_{\mathrm{x}}$ and $T_{\mathrm{e}}$ are correlated (Fig. 4b, e), and the $\Gamma-T_{\mathrm{e}}$ dependency is also reversed. In the $\tau-$ $T_{\mathrm{e}}$ diagram, we find a range of $\tau$ values $(\tau \approx[2.5-4])$ for which $T_{\mathrm{e}}$ displays a roughly constant value $(\sim 40 \mathrm{keV})$. Below $\tau \lesssim 2.5$ $T_{\mathrm{e}}$ and $\tau$ are anti-correlated (Fig. 4f).

For spectra softer than $\Gamma \gtrsim 2.4$ the spectral fits using NTHCOMP do not provide constrained electron temperatures. These soft spectra are detected at the highest $\left(\gtrsim 20 \times 10^{-8} \mathrm{erg} \mathrm{cm}^{-2} \mathrm{~s}^{-1}\right)$ and lowest $\left(\$ 2 \times 10^{-8} \mathrm{erg} \mathrm{cm}^{-2} \mathrm{~s}^{-1}\right) F_{\mathrm{x}}$ values. When detected at the highest fluxes, they occupy regions in the $F_{\mathrm{x}}-\Gamma$ diagram (Fig. 4a) that are reminiscent of the HID ultra-luminous state (Motta et al. 2014).

Finally, we also observe that the spectra detected during $\mathrm{X}$-ray plateaus occupy separate regions, in all these diagrams, which are distinct from the Comptonized branches; this confirms our classification of these spectra in a separate category. We call these regions plateau branch(es).

\subsection{Fits to the plateau spectra using variable absorption}

To study the poor fitting spectra detected during X-ray plateaus, we also considered the possibility that these were the result of obscuration of the primary $\mathrm{X}$-ray emitting region. Absorption by Compton-thick material $\left(N_{\mathrm{H}} \gtrsim 10^{24} \mathrm{~cm}^{-2}\right)$ can substantially reduce the source flux for energies up to $\sim 30$ $40 \mathrm{keV}$, and also change the spectral slope and produce a global flux reduction by a factor 10 in the $40-300 \mathrm{keV}$ energy range (Murphy \& Yaqoob 2009). Joint spectral fits to simultaneous Swift/XRT and INTEGRAL/JEMX+ISGRI spectra during the X-ray plateau around MJD 57195 by Motta et al. (2017) already showed that the broadband spectrum of V404 Cyg around that period was compatible with heavily absorbed Comptonized emission $\left(N_{\mathrm{H}} \sim 1-3 \times 10^{24} \mathrm{~cm}^{-2}\right)$ with a prominent scattered component.

For the above reasons, we performed two additional fitting runs using COMPPS. In the first fitting run, we left $N_{\mathrm{H}}$ as a free parameter. The results of these fits are presented in Fig. 5. In the second fitting run we also allowed the Compton reflection amplitude parameter in COMPPS to vary freely. The results obtained in these fits are presented in Fig. 6. In both cases, we compared the results obtained in these fits against those obtained using a model where the absorption was fixed to interstellar values, as described in the previous sections. We computed the BIC for each fit, and again applied the $\triangle \mathrm{BIC}>6$ criterium to select the 

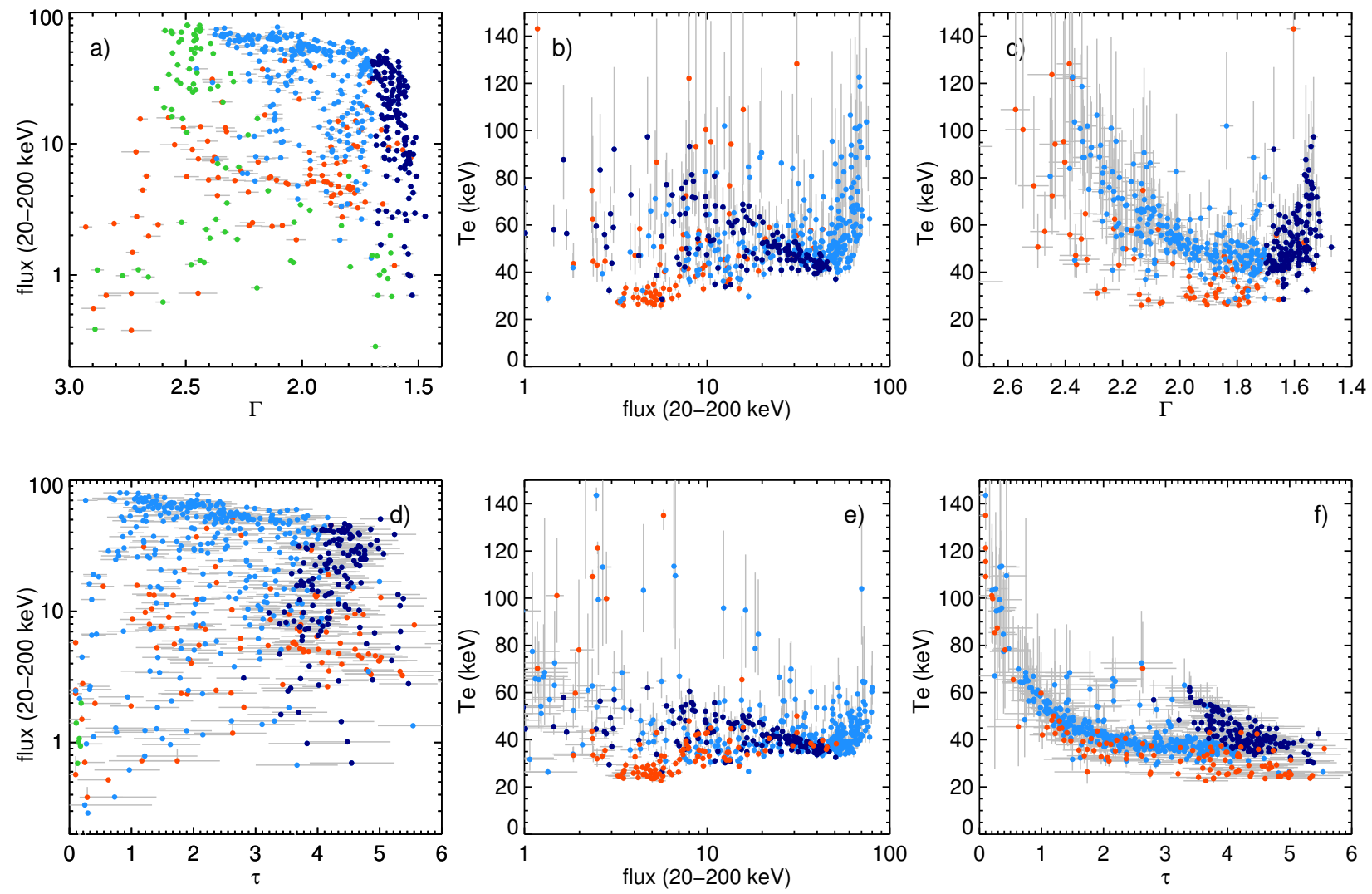

Fig. 4. Relations between the parameters derived from our spectral fits, via the NTHCOMP (panels a)-c)) and comPPS models (panels d)-f)). Green, blue and red symbols are used to highlight the best-fitting model, according to our model selection criteria (Sect. 2.2). Blue: comptonization models with constrained $T_{\mathrm{e}}$ (Fig. 1a) further divided into hard ( $\Gamma \leq 1.7$; dark blue) and soft spectra ( $\Gamma>1.7$; light blue). Green: Comptonization models with unconstrained $T_{\mathrm{e}}$ (Fig. 1b). Red: $p$-value $<0.05$ fits. (Fig. 1c). Panel a) $F_{\mathrm{x}}-\Gamma$ diagram. We find a hard branch (dark blue points) where $F_{\mathrm{x}}$ and $\Gamma$ are correlated, similar to the hard state in the BHB HID. At the highest fluxes in the outburst, we find the soft flaring branch (light blue points), similar to the BHB HID intermediate states. We also identify the softest spectra in our sample $(\Gamma \gtrsim 2.4)$ with unconstrained $T_{\mathrm{e}}$ with a tentative ultra-luminous state (green points). The different branches also occupy characteristic regions in panel d) $F_{\mathrm{x}}-\tau$ diagram, panels b, e), $F_{\mathrm{x}}-T_{\mathrm{e}}$ diagrams, panel c) $\Gamma-T_{\mathrm{e}}$ diagram and panel f) $\tau-T_{\mathrm{e}}$ diagram. The various parameter correlations are described in detail in Sect. 3.3 .

model better describing the data. Also, the $p$-value of the fit with respect to the data was verified in these new fits.

For the spectra obtained during the X-ray plateaus, the $\triangle \mathrm{BIC}$ model selection criterium favours a Comptonized model with $N_{\mathrm{H}}$ values in excess of the interstellar one $\left(N_{\mathrm{H}} \gtrsim 5 \times 10^{24} \mathrm{~cm}^{-2}\right.$; see Figs. 5, 6) regardless of whether reflection is considered or not. However, $N_{\mathrm{H}}$ is constrained better when no reflection is considered (see Fig. 5). The validity of the models is supported by the $p$-value of the fit with respect to the data, which is now $p$-value $\geq 0.05$ for the fits to the plateau spectra when the absorption is left to vary freely, with or without reflection. During X-ray flares, the BIC model selection favours a Comptonized model where the absorption is fixed to the interstellar values. The energy range analysed in this work (20-200 keV) only allows firm constraints on the most extreme $N_{\mathrm{H}}$ values $\left(N_{\mathrm{H}} \gtrsim 5 \times 10^{24} \mathrm{~cm}^{-2}\right)$. Although we observe an evolution of the derived $N_{\mathrm{H}}$ values in anti-correlation with the flux evolution (i.e. $N_{\mathrm{H}}$ reaches the highest values during the $\mathrm{X}$-ray plateaus and decreases during the X-ray flares).

Using solely the 20-200 keV energy range of our spectra, we cannot simultaneously constrain $N_{\mathrm{H}}$ and the reflection contribution to the source spectra (quantified using the $R$ parameter in COMPPS, Fig. 6e), as shown by the large uncertainties in the determination of both parameters (Figs. 6d, e). The BIC model selection criterium favours a Comptonized model with variable column density over a Comptonized model with variable column density and variable reflection. Only by extending the fitting range to lower energies we will be able to model simultaneously the absorption and reflection parameters in our fits.

Finally, we note that with the spectral fits presented in this section, the peculiar increase of $\tau$, and simultaneous decrease of $T_{\mathrm{e}}$ derived during the X-ray plateaus largely disappears, (see Figs. 2, 5, 6) suggesting that the systematic changes of these parameters were the result of inaccurate modelling.

\section{Discussion}

The light curve of V404 Cyg during the June 2015 outburst does not display the typical features of the standard BHB light curves (e.g. Chen et al. 1997; Remillard \& McClintock 2006). Similarly, the soft X-ray spectra of V404 Cyg are remarkably different from the spectra of other BHBs, mostly owing to extreme intrinsic absorption (Motta et al. 2017) also seen in the 1989 outburst (Życki et al. 1999). However, when we look at the source spectra in hard X-rays (above $20 \mathrm{keV}$ ), we find some similarities between V404 Cyg and other BHBs.

\subsection{Hard branch}

We have identified a hard branch in the $F_{X}-\Gamma$ diagram of V404 Cyg that is reminiscent of the hard state branch of the 

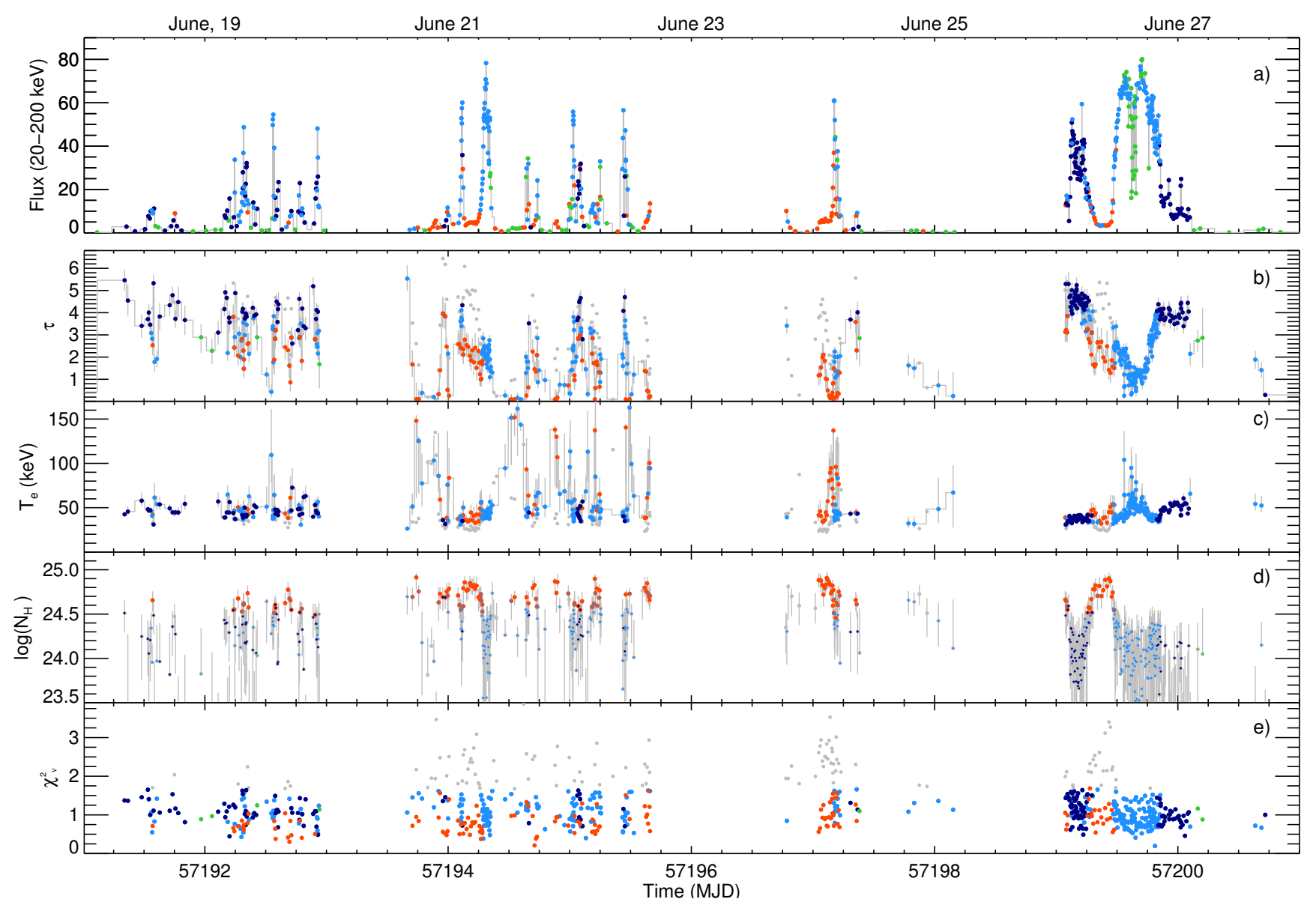

Fig. 5. Time evolution of the flux and spectral parameters of V404 Cyg during the June 2015 flaring episodes. Green and blue symbols are unchanged with respect to Fig. 2. Red symbols highlight the parameters obtained fitting the data with a Comptonized model (ComPPS) modified by variable absorption. The BIC model selection (see text) favours this model only for the spectra detected during the X-ray plateaus. For comparison, we also show the parameters obtained in the fits to these spectra fixing the absorption to interstellar values (grey symbols in panels b), c) and e). Panel a) source flux (20-200 keV) in units of $10^{-8} \mathrm{erg} \mathrm{cm}^{-2} \mathrm{~s}^{-1}$. Panel b) optical depth, $\tau$, Panel c) electron temperature, $T_{\mathrm{e}}$. Panel d) $N_{\mathrm{H}}$ values obtained for the whole data set when leaving $N_{\mathrm{H}}$ as a free parameter; only when $N_{\mathrm{H}} \gtrsim 10^{24} \mathrm{~cm}^{-2}$ modifies substantially the spectral shape in the IBIS/ISGRI energy range, can this value be properly constrained in our spectral fits. This happens during the X-ray plateaus. Panel e) $\chi_{v}^{2}$ value for every fit.

BHB HID (Homan et al. 2001; Belloni 2004; Fender et al. 2004; Dunn et al. 2010; Belloni \& Motta 2016). The hard branch is occupied by the hardest spectra in our sample $(\Gamma \leq 1.7)$. In the hard branch, the spectrum of V404 Cyg gradually softens and $T_{\mathrm{e}}$ decreases from $T_{\mathrm{e}} \sim 80 \mathrm{keV}$ or unconstrained (NTHCOMP) down to about $T_{\mathrm{e}} \sim 40 \mathrm{keV}$ as the flux increases (Fig. $4 \mathrm{a}$, $\mathrm{d}, \mathrm{b}, \mathrm{e})$. This $F_{\mathrm{x}}-T_{\mathrm{e}}$ anti-correlation has been observed by Natalucci et al. (2015) and Roques et al. (2015) in the analysis of IBIS/ISGRI and SPI data obtained during rev. 1554 (EPOCH 1) and by Jenke et al. (2016) using Fermi/GBM data. Similar anticorrelations have also been found in other BHBs in the hard state (Esin et al. 1998; Wardziński et al. 2002; Rodriguez et al. 2003; Motta et al. 2009; Kajava et al. 2016), supporting our identification of the hard branch with the hard state of prototypical BHBs (Belloni \& Motta 2016). The $F_{\mathrm{x}}-T_{\mathrm{e}}$ anti-correlation in the hard branch can be explained by the truncated disk/hot inner flow model (Esin et al. 1997; Mayer \& Pringle 2007), which assumes that at low luminosities the accretion disk is truncated at distances between a few tens and a few thousand gravitational radii from the $\mathrm{BH}$, and only a small fraction of disk photons reach the hot flow/comptonizing medium. The X-ray spectrum would then be produced by pure synchrotron self-Compton emission (SSC) in an hybrid (thermal plus non-thermal) Comptonizing medium (Poutanen \& Vurm 2009; Malzac \& Belmont 2009; Veledina et al. 2011). V404 Cyg may be in this regime at fluxes below $\sim 10^{-8} \mathrm{erg} \mathrm{cm}^{-2} \mathrm{~s}^{-1}$, where we measure $T_{\mathrm{e}}$ in the range $60-80 \mathrm{keV}$ (or unconstrained). As the accretion rate increases, the inner radius of the accretion disk moves inwards, closer to the $\mathrm{BH}$, and a growing number of soft seed photons from the accretion disk enter the Comptonizing medium, gradually cooling down the population of thermal electrons (Poutanen \& Vurm 2009; Malzac \& Belmont 2009; Veledina et al. 2011). Electron cooling results in softer Comptonized spectra (Done et al. 2007). The electron cooling could cause the observed $F_{\mathrm{x}}-T_{\mathrm{e}}$ anti-correlation and gradual spectral softening in the hard branch. Observations of GX 339-4 (Wardziński et al. 2002) and GRO J1655-40 (Joinet et al. 2008) in the hard state result in $T_{\mathrm{e}}$ values that are comparable to our measurements. However, these systems displayed lower $\tau$ values $(\tau \approx 2.5)$ than those measured for V404 Cyg $(\tau \approx[3-5.5])$ which suggests that we are dealing with an optically thicker Comptonizing medium. The $\tau$ values we derive are comparable to those found during the 1989 outburst of V404 Cyg $(\tau \approx 6$; Życki et al. 1999). As the optical depth is expected to scale linearly with the mass accretion rate (Różańska \& Czerny 2000), the presence of an optically thick Comptonizing medium may 

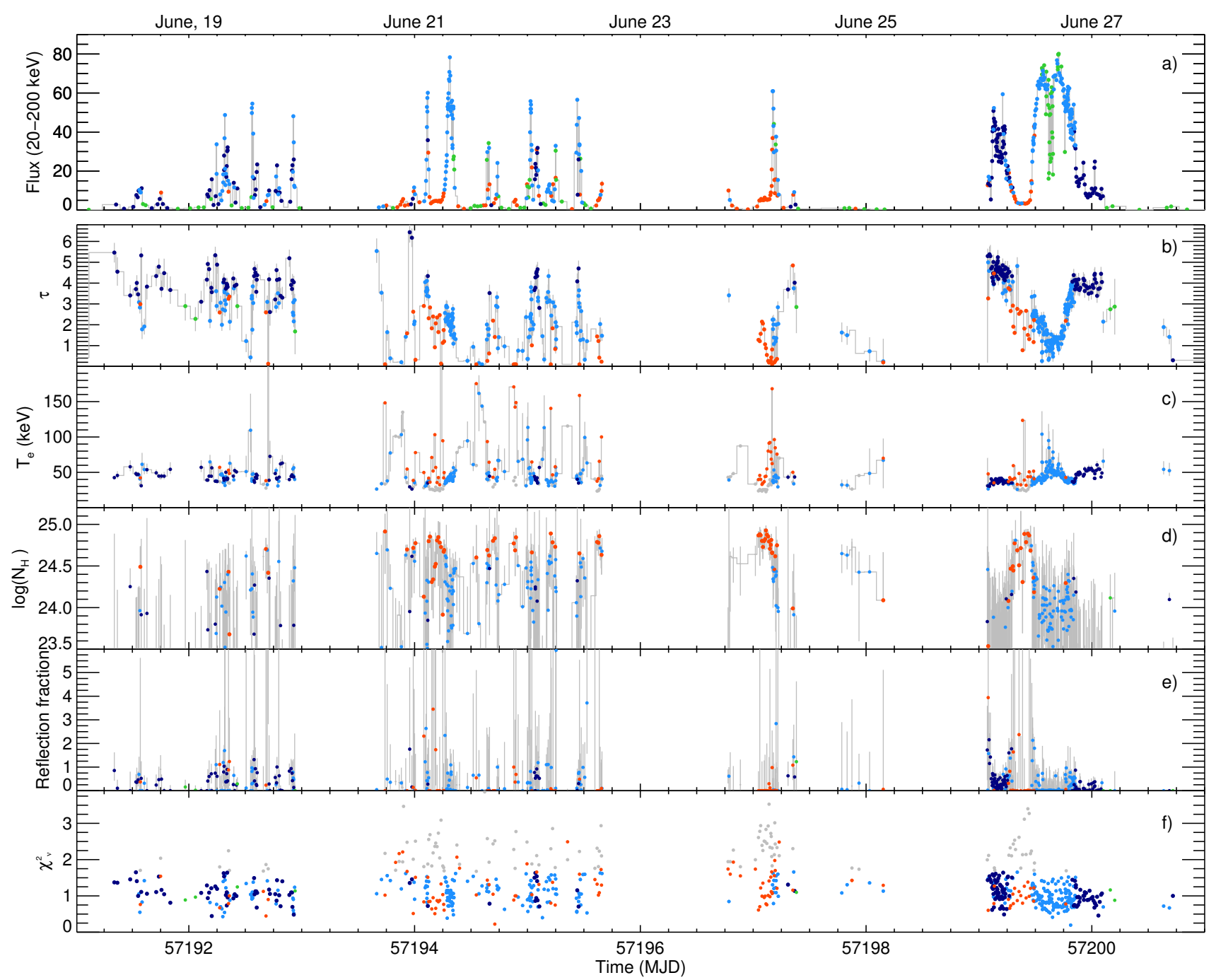

Fig. 6. Same as Fig. 5, but for a Comptonized model (COMPPS) modified by variable absorption and reflection. Panel a) source flux (20-200 keV) in units of $10^{-8} \mathrm{erg} \mathrm{cm}^{-2} \mathrm{~s}^{-1}$. Panel b) optical depth, $\tau$, Panel c) electron temperature, $T_{\mathrm{e}}$. Panel d) $N_{\mathrm{H}}$ values obtained for the whole data set when leaving $N_{\mathrm{H}}$ as a free parameter. Panel e) Reflection fraction (Parameter R in COMPPS). Panel f) $\chi_{v}^{2}$ value for each fit.

be connected to V404 Cyg emitting closer to the Eddington limit than other BHBs in the hard state.

\subsection{Soft flaring branch}

In the soft flaring branch the spectrum still softens as the flux increases, but most of the parameter dependencies are reversed with respect to the hard branch, suggesting a change in the hard X-ray production mechanism: as $F_{\mathrm{x}}$ increases, the optical depth of the Comptonizing medium decreases and the spectrum softens (Fig. 4c), while the electron temperature increases (Fig. 4b, e). These parameter relations are similar to those observed during hard to soft state transitions in other BHBs (Phlips et al. 1996; Esin et al. 1998; Joinet et al. 2008; Motta et al. 2009; Del Santo et al. 2013), suggesting that the soft flaring branch may correspond to the BHB intermediate state. The decrease in $\tau$ observed during hard to soft state transitions is consistent with the material in the Comptonizing medium condensing into a disk, or being ejected into an outflow (Malzac 2016). The detection of a cut-off in the spectrum is indicative of a significant fraction of thermal electrons in the Comptonizing medium. The parameter Te increases as the flux progressively increases, suggesting that the injection of external (disk) photons, which cooled down the electron cloud in the hard branch might have ceased or its cooling effect on the electron cloud is negligible.

The steepest spectra in our sample with $\Gamma \gtrsim 2.4$ (or $\tau \lesssim 1.0$ ) are those where $T_{\mathrm{e}}$ is not constrained (NTHCOMP). Fits to these data using COMPPS provide extremely low $\tau$ and high $T_{\mathrm{e}}$ values. When the optical depth is low, the particles do not have time to thermalize between re-accelerations, and the electron distribution resembles the power-law injection function that could originate from magnetic reconnection or shock acceleration (Veledina et al. 2011; Del Santo et al. 2013). These spectra are detected at epochs bracketed by the largest measured $T_{\mathrm{e}}$ values $(\gtrsim 80 \mathrm{keV})$, so it is likely that they are caused by the electron population becoming simultaneously hotter and/or progressively less thermal. When detected at the highest fluxes $\left(\gtrsim 20 \times 10^{-8} \mathrm{erg} \mathrm{cm}^{-2} \mathrm{~s}^{-1}\right)$, the $\Gamma \gtrsim 2.4$ spectra occupy a region in the $F_{\mathrm{x}}-\Gamma$ diagram (Fig. 4a) that is reminiscent of the HID ultra-luminous state (Dunn et al. 2010), which suggests that these spectra could be analogous to the ultra-luminous state of GX 339-4 (Miyamoto et al. 1991; Kubota \& Done 2016), GS 1124-68 
(Miyamoto et al. 1993), XTE J1550-564 (Sobczak et al. 1999b; Kubota \& Makishima 2004; Hjalmarsdotter et al. 2016), GRO J1655-40 (Sobczak et al. 1999a; Joinet et al. 2008), or 4 U 1630-47 (Abe et al. 2005). In such a state the spectrum is a composite of a strong disk and a steep prominent Comptonized tail, with no cut-off at high energies, which may extend up to $\sim 1 \mathrm{MeV}$ (Kubota \& Done 2016).

When detected at the lowest fluxes, these $\Gamma \gtrsim 2.4$ spectra could be analogous to the soft state spectra of some BHB where faint non-thermal hard X-ray tails are detected (Zdziarski et al. 2017).

\subsection{X-ray plateaus}

We have observed X-ray plateaus characterized by roughly constant fluxes $\left(F_{\mathrm{x}} \sim 5 \times 10^{-8} \mathrm{erg} \mathrm{cm}^{-2} \mathrm{~s}^{-1}\right)$ during periods lasting several hours. Often the plateaus are observed in between two or several flares (see Figs. 2, A.1). Furthermore, we only detect $\mathrm{X}$-ray plateaus in epochs 2 and 3, when the source is predominantly in the soft flaring branch, where the spectrum is softer $(\Gamma>1.7)$. We observed that the statistically worse fits $\left(\chi_{v}^{2}>1.8\right.$; see Fig. 2) tend to happen during these X-ray plateaus if we fix $N_{\mathrm{H}}$ to the interstellar value in the direction of the source. The statistics are improved when allowing $N_{\mathrm{H}}$ to vary in our fits $\left(\chi_{v}^{2} \sim 1\right.$; see Figs. 5, 6). In this case we derive $N_{\mathrm{H}}$ values $\left(N_{\mathrm{H}} \gtrsim 5 \times 10^{24} \mathrm{~cm}^{-2}\right)$ two orders of magnitude in excess of the interstellar $N_{\mathrm{H}}$ value in the direction of the source $\left(N_{\mathrm{H}} \sim 0.8 \times 10^{22} \mathrm{~cm}^{-2}\right)$, which suggests that intrinsic absorption by Compton-thick material distorts the source spectrum and results in the spectral shape observed during the X-ray plateaus (see Fig. 1c). Owing to the limited energy range available for this analysis, our fits cannot constrain simultaneously $N_{\mathrm{H}}$ and the fraction of X-ray photons reprocessed by the Compton-thick material contributing to the source spectra (i.e. the reflection fraction in COMPPS). Also, if $N_{\mathrm{H}}$ is allowed to vary in our fits, the spectral parameters $T_{\mathrm{e}}$ and $\tau$ display values that are consistent with measurements during nearly contemporaneous flares.

In previous studies, Natalucci et al. (2015), Roques et al. (2015), and Jenke et al. (2016) inferred very high seed photon temperatures $\sim 6-7 \mathrm{keV}$, which they attributed to the jet. Our results show that the source spectra can also be modelled using lower seed photons temperatures (consistent with Swift/XRT results; Motta et al. 2017) and strong local absorption.

\subsection{Flaring activity}

Although $N_{\mathrm{H}}$ cannot be properly constrained during the X-ray flares, when lower $N_{\mathrm{H}}$ values are derived, $N_{\mathrm{H}}$ is seen to vary in anti-correlation with the evolving $\mathrm{X}$-ray flux over the whole data set analysed here (Fig. 5c). The fact that we derive $N_{\mathrm{H}} \sim 5 \times$ $10^{24} \mathrm{~cm}^{-2}$ only during the X-ray plateaus, and clearly lower $N_{\mathrm{H}}$ values in the adjacent flares, suggests that the dramatic intensity drops observed during these plateaus may be partially caused by obscuration of the central source. The obscuring material can be some outflowing material, the outer regions of a Comptonthick accretion disk, or a combination of both. Thus, the apparent flaring activity may actually be the result of a clumpy Comptonthick obscuring material becoming occasionally Compton-thin and allowing the source photons to reach the observer. The fast flare rise and decay times $(\approx 30 \mathrm{~min}$, see Fig. A.1) may actually be related to varying partial obscuration of the central source, as previously suggested by Życki et al. (1999), who measured $N_{\mathrm{H}}$ variability on timescales of minutes during the 1989 outburst.
Perhaps also for the same reason we find a lot of scatter in the correlations between $F_{\mathrm{x}}$ and the source spectral parameters $(\Gamma, \tau$, $T_{\mathrm{e}}$ ), while there is much less scatter in the various parameter correlations ( $\Gamma-T_{\mathrm{e}}, \tau-T_{\mathrm{e}}$; see Fig. 4$)$.

\section{Summary of results and conclusions}

We have fit the 20-200 keV IBIS/ISGRI spectra of V404 Cyg during the June 2015 outburst using two thermal Comptonization models (NTHCOMP and COMPPS). For the first time we continuously measured the evolution of the properties of the Comptonizing medium during an outburst rise and decay. We find that the system evolves through the same $\Gamma-T_{\mathrm{e}}, \tau-T_{\mathrm{e}}$ and $F_{\mathrm{x}}-T_{\mathrm{e}}$ paths when the outburst rises or decays. We identified two clear spectral branches in the $F_{\mathrm{x}}-\Gamma$ diagram, which display characteristic parameter relations: a hard branch and a soft flaring branch.

In the hard branch, V404 Cyg shows a hard $(\Gamma \leq 1.7)$ thermal Comptonized spectrum, which slowly softens as the flux increases. In the hard branch, $\tau$ is correlated with $F_{\mathrm{x}}$ while $T_{\mathrm{e}}$ is anti-correlated with $F_{\mathrm{x}}$ and $\Gamma$. Similar parameter correlations have been observed in other BHBs in the hard state, suggesting that the hard branch could correspond to the HID hard state. The observed parameter evolution can be explained in terms of thermal Comptonization of soft seed photons by a hot electron cloud in the vicinity of the $\mathrm{BH}$. The $F_{\mathrm{x}}-T_{\mathrm{e}}$ anti-correlation could result from the electron population progressively cooling down as the accretion disk moves closer to the $\mathrm{BH}$ and more disk photons enter the Comptonizing medium.

In the soft flaring branch V404 Cyg shows a soft, thermal Comptonized spectrum, $(\Gamma>1.7)$, which softens as the flux increases. In the soft flaring branch $T_{\mathrm{e}}$ and $F_{\mathrm{x}}$ are correlated, while $F_{\mathrm{X}}$ and $\tau$ are anti-correlated. The parameter correlations are consistent with those observed during hard to soft state transitions in other sources, suggesting that these data could correspond to the intermediate state or occasionally the ultra-luminous state. The observed $T_{\mathrm{e}}-F_{\mathrm{x}}$ correlation is compatible with the predictions of SSC-models.

We also found a plateau branch where Comptonization models fail to describe the source spectra if $N_{\mathrm{H}}$ is fixed to the interstellar values in the direction of the source. The fits to these spectra improve when we leave the absorption to vary freely. In this case we derive $N_{\mathrm{H}}$ values $\left(N_{\mathrm{H}} \gtrsim 5 \times 10^{24} \mathrm{~cm}^{-2}\right)$ which suggest that intrinsic absorption by Compton-thick material results in the spectral shape observed during the X-ray plateaus. The obscuring material can be some outflowing material, a clumpy Compton-thick accretion disk, or a combination of both. Thus, we propose that the observed dramatic flaring activity seen at hard X-rays may not only be due to intrinsic source variability, but can partly result from obscuration of the central source by Compton-thick material. The system inclination of $67^{\circ}$ may be a key parameter in the observation of such phenomenology that is not observed in other sources seen at lower or higher inclination angles.

Acknowledgements. The authors would like to thank the anonymous referee for useful comments that contributed to improving the paper. J.J.E.K. was supported by Academy of Finland grants 268740 and 295114, and the ESA research fellowship program. S.E.M. acknowledges support from the Faculty of the European Space Astronomy Centre (ESAC).

\section{References}

Abe, Y., Fukazawa, Y., Kubota, A., Kasama, D., \& Makishima, K. 2005, PASJ, 57,629

Arnaud, K. A. 1996, in Astronomical Data Analysis Software and Systems V, eds. G. H. Jacoby, \& J. Barnes, ASP Conf. Ser., 101, 17 
Barthelmy, S. D., \& Sbarufatti, B. 2015, GRB Coordinates Network, 17963 Belloni, T. 2004, Nucl. Phys. B Proc. Suppl., 132, 337

Belloni, T. M., \& Motta, S. E. 2016, in Astrophysics of Black Holes: From Fundamental Aspects to Latest Developments, ed. C. Bambi, Astrophys Space Sci. Libr., 440, 61

Beloborodov, A. M. 1999, in High Energy Processes in Accreting Black Holes, eds. J. Poutanen, \& R. Svensson, ASP Conf. Ser., 161, 295

Casares, J., Charles, P. A., \& Naylor, T. 1992, Nature, 355, 614

Chen, W., Shrader, C. R., \& Livio, M. 1997, ApJ, 491, 312

Courvoisier, T. J.-L., Walter, R., Beckmann, V., et al. 2003, A\&A, 411, L53

Del Santo, M., Malzac, J., Belmont, R., Bouchet, L., \& De Cesare, G. 2013 MNRAS, 430, 209

Done, C., Gierliński, M., \& Kubota, A. 2007, A\&ARv, 15, 1

Droulans, R., Belmont, R., Malzac, J., \& Jourdain, E. 2010, ApJ, 717, 1022

Dunn, R. J. H., Fender, R. P., Körding, E. G., Belloni, T., \& Cabanac, C. 2010, MNRAS, 403, 61

Ebisawa, K., Ogawa, M., Aoki, T., et al. 1994, PASJ, 46, 375

Eikenberry, S. S., Dallilar, Y., Garner, A., et al. 2016, AAS/High Energy Astrophysics Division, HEAD Meeting 15, 109.01

Esin, A. A., McClintock, J. E., \& Narayan, R. 1997, ApJ, 489, 865

Esin, A. A., Narayan, R., Cui, W., Grove, J. E., \& Zhang, S.-N. 1998, ApJ, 505 854

Fender, R. P., Belloni, T. M., \& Gallo, E. 2004, MNRAS, 355, 1105

Ferrigno, C., Bozzo, E., Boissay, R., Kuulkers, E., \& Kretschmar, P. 2015, ATel, 7731

Gandhi, P., Littlefair, S. P., Hardy, L. K., et al. 2016, MNRAS, 459, 554

Gierliński, M., Zdziarski, A. A., Poutanen, J., et al. 1999, MNRAS, 309, 496

Hjalmarsdotter, L., Axelsson, M., \& Done, C. 2016, MNRAS, 456, 4354

Homan, J., Wijnands, R., van der Klis, M., et al. 2001, ApJS, 132, 377

Jenke, P. A., Wilson-Hodge, C. A., Homan, J., et al. 2016, ApJ, 826, 37

Joinet, A., Jourdain, E., Malzac, J., et al. 2007, ApJ, 657, 400

Joinet, A., Kalemci, E., \& Senziani, F. 2008, ApJ, 679, 655

Kajava, J. J. E., Veledina, A., Tsygankov, S., \& Neustroev, V. 2016, A\&A, 591, A66

Kass, R., \& Raftery, A. 1995, Journal of the American Statistical Association, 90, 773

Khargharia, J., Froning, C. S., \& Robinson, E. L. 2010, ApJ, 716, 1105

Kimura, M., Isogai, K., Kato, T., et al. 2016, Nature, 529, 54

Kubota, A., \& Done, C. 2016, MNRAS, 458, 4238

Kubota, A., \& Makishima, K. 2004, ApJ, 601, 428

Kuulkers, E. 2015, ATel, 7758

Lebrun, F., Leray, J. P., Lavocat, P., et al. 2003, A\&A, 411, L141

Loh, A., Corbel, S., Dubus, G., et al. 2016, MNRAS, 462, L111

Makino, F. 1989, IAU Circ., 4782,

Malzac, J. 2016, Astron. Nachr., 337, 391

Malzac, J., \& Belmont, R. 2009, MNRAS, 392, 570

Malzac, J., Petrucci, P. O., Jourdain, E., et al. 2006, A\&A, 448, 1125

Marsden, B. G. 1989, IAU Circ., 4783

Mayer, M., \& Pringle, J. E. 2007, MNRAS, 376, 435

McConnell, M. L., Zdziarski, A. A., Bennett, K., et al. 2002, ApJ, 572, 984

Miller-Jones, J. C. A., Jonker, P. G., Dhawan, V., et al. 2009, ApJ, 706, L230
Miyamoto, S., Kimura, K., Kitamoto, S., Dotani, T., \& Ebisawa, K. 1991, ApJ, 383,784

Miyamoto, S., Iga, S., Kitamoto, S., \& Kamado, Y. 1993, ApJ, 403, L39

Motta, S., Belloni, T., \& Homan, J. 2009, MNRAS, 400, 1603

Motta, S., Homan, J., Muñoz Darias, T., et al. 2012, MNRAS, 427, 595

Motta, S. E., Belloni, T. M., Stella, L., Muñoz-Darias, T., \& Fender, R. 2014, MNRAS, 437, 2554

Motta, S. E., Kajava, J. J. E., Sánchez-Fernández, C., Giustini, M., \& Kuulkers, E. 2017, MNRAS, 468, 981

Muñoz-Darias, T., Casares, J., Mata Sánchez, D., et al. 2016, Nature, 534, 75

Murphy, K. D., \& Yaqoob, T. 2009, MNRAS, 397, 1549

Narayan, R., \& Yi, I. 1995, ApJ, 452, 710

Natalucci, L., Fiocchi, M., Bazzano, A., et al. 2015, ApJ, 813, L21

Negoro, H., Matsumitsu, T., Mihara, T., et al. 2015, ATel, 7646

Oosterbroek, T., van der Klis, M., Vaughan, B., et al. 1996, A\&A, 309, 781

Phlips, B. F., Jung, G. V., Leising, M. D., et al. 1996, ApJ, 465, 907

Poutanen, J., \& Svensson, R. 1996, ApJ, 470, 249

Poutanen, J., \& Veledina, A. 2014, Space Sci. Rev., 183, 61

Poutanen, J., \& Vurm, I. 2009, ApJ, 690, L97

Remillard, R. A., \& McClintock, J. E. 2006, ARA\&A, 44, 49

Richter, G. A. 1989, Information Bulletin on Variable Stars, 3362

Rodriguez, J., Corbel, S., \& Tomsick, J. A. 2003, ApJ, 595, 1032

Rodriguez, J., Cadolle Bel, M., Alfonso-Garzón, J., et al. 2015, A\&A, 581, L9

Roques, J.-P., Jourdain, E., Bazzano, A., et al. 2015, ApJ, 813, L22

Różańska, A., \& Czerny, B. 2000, A\&A, 360, 1170

Schwarz, G. 1978, Annals of statistics, 6, 461

Segreto, A., Del Santo, M., D’Aí, A., et al. 2015, ATel, 7755

Shakura, N. I., \& Sunyaev, R. A. 1973, A\&A, 24, 337

Shapiro, S. L., Lightman, A. P., \& Eardley, D. M. 1976, ApJ, 204, 187

Siegert, T., Diehl, R., Greiner, J., et al. 2016, Nature, 531, 341

Sivakoff, G. R., Bahramian, A., Altamirano, D., et al. 2015, ATel, 7959

Sobczak, G. J., McClintock, J. E., Remillard, R. A., Bailyn, C. D., \& Orosz, J. A. 1999a, ApJ, 520, 776

Sobczak, G. J., McClintock, J. E., Remillard, R. A., et al. 1999b, ApJ, 517, L121

Sunyaev, R. A., \& Truemper, J. 1979, Nature, 279, 506

Tanaka, Y. 1989, in Two Topics in X-Ray Astronomy, Volume 1: X Ray Binaries. Volume 2: AGN and the X Ray Background, eds. J. Hunt, \& B. Battrick, ESA SP, 296

Tetarenko, A., Sivakoff, G. R., Young, K., Wouterloot, J. G. A., \& Miller-Jones, J. C. 2015, ATel, 7708

Veledina, A., Vurm, I., \& Poutanen, J. 2011, MNRAS, 414, 3330

Walton, D., Harrison, F., Forster, K., et al. 2015, ATel, 7752

Walton, D. E. 2016, AAS/High Energy Astrophysics Division, 15, 109.08

Wardziński, G., Zdziarski, A. A., Gierliński, M., et al. 2002, MNRAS, 337, 829

Winkler, C., Courvoisier, T. J.-L., Di Cocco, G., et al. 2003, A\&A, 411, L1

Younes, G. 2015, GRB Coordinates Network, 17932

Zdziarski, A. A., Johnson, W. N., \& Magdziarz, P. 1996, MNRAS, 283, 193

Zdziarski, A. A., Lubiński, P., \& Sikora, M. 2012, MNRAS, 423, 663

Zdziarski, A. A., Malyshev, D., Chernyakova, M., \& Pooley, G. G. 2017, MNRAS, submitted

Życki, P. T., Done, C., \& Smith, D. A. 1999, MNRAS, 309, 561 


\section{Appendix A: Closer view to some flares}
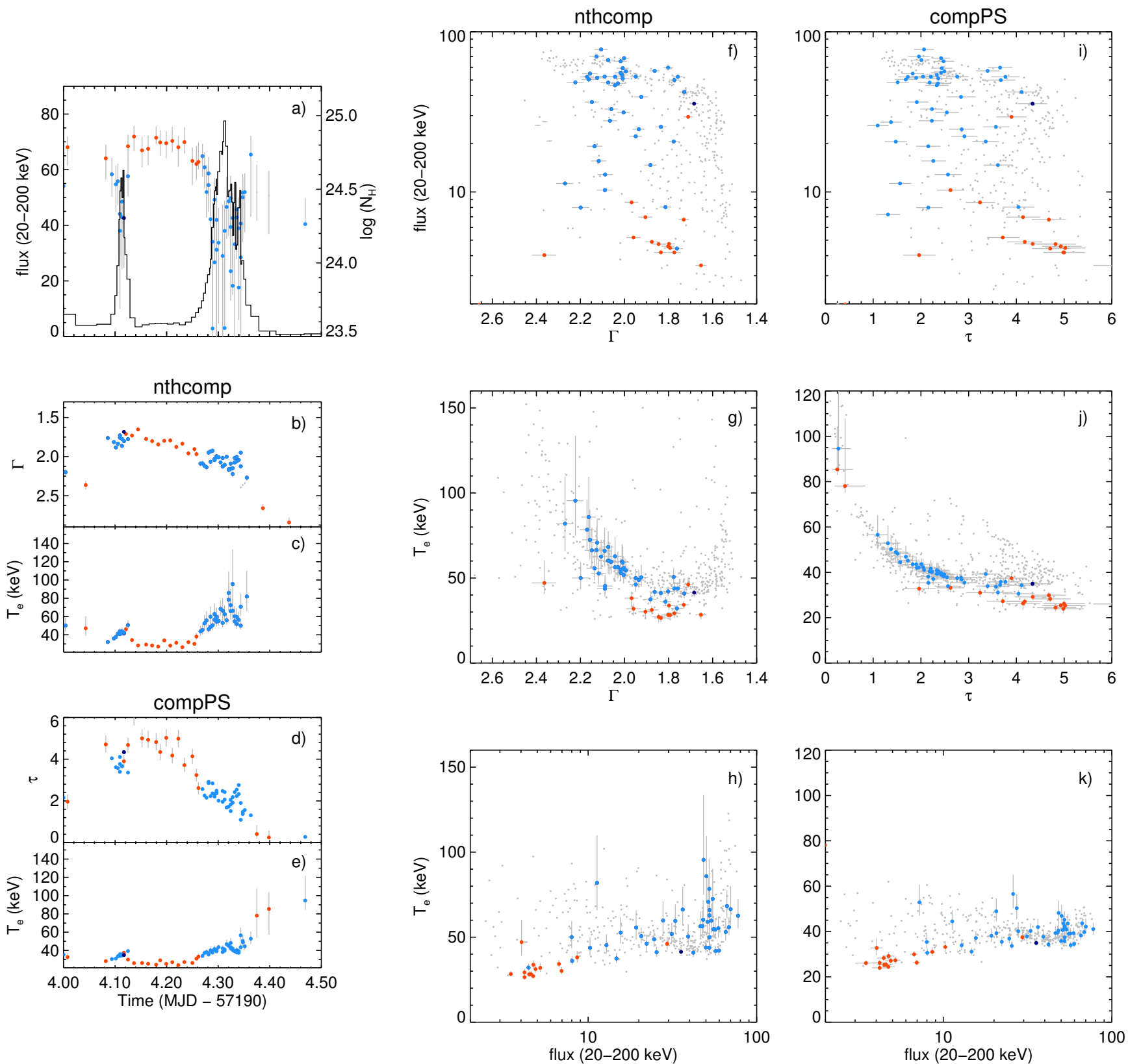

Fig. A.1. Close view of the properties of the X-ray plateau detected on MJD 57 194.0-57 194.4, derived fitting the source spectra with Comptonization models (NTHCOMP and COMPPS). Panel a) time evolution of the source flux, $F_{\mathrm{x}}$, in the $20-200 \mathrm{keV}$ energy range $\left(\times 10^{-8} \mathrm{erg} \mathrm{cm}^{-2} \mathrm{~s}^{-1}\right)$ in comparison with the $N_{\mathrm{H}}$ evolution, where $N_{\mathrm{H}}$ was derived by leaving it as a free parameter in our fits, as described in Sect. 3.4. The plateau was characterized by a roughly constant flux $F_{\mathrm{x}} \sim 5 \times 10^{-8} \mathrm{erg} \mathrm{cm}^{-2} \mathrm{~s}^{-1}$ and was interrupted by two big flares around MJD 57194.1 and 57194.3 with peak fluxes of $F_{\mathrm{x}} \sim 60$ and $80 \times 10^{-8} \mathrm{erg} \mathrm{cm}^{-2} \mathrm{~s}^{-1}$. We observed roughly constant $N_{\mathrm{H}}$ values during the X-ray plateau $\left(N_{\mathrm{H}} \sim 5 \times 10^{24} \mathrm{~cm}^{-2}\right)$. Systematically lower $N_{\mathrm{H}}$ values were measured during the two X-ray flares. However, $N_{\mathrm{H}}$ cannot be properly constrained by our fits to the flare spectra. Panels b)-e) time evolution of the spectral parameters derived for this period. Panels f)-h) relations between the various NTHCOMP spectral parameters during this period. Panels i)-k) relations between the various compPS spectral parameters during this period. For reference, we also show in panels f)-k) the complete dataset analysed in this work (grey points). The parameters derived during the peaks of the flares occupy the soft flaring branch, (see Sect. 3.3), while the flare rise and decays, which are likely affected by partial obscuration, occupy intermediate regions between the soft flaring branch and the plateau branch in the panels f) and i). 

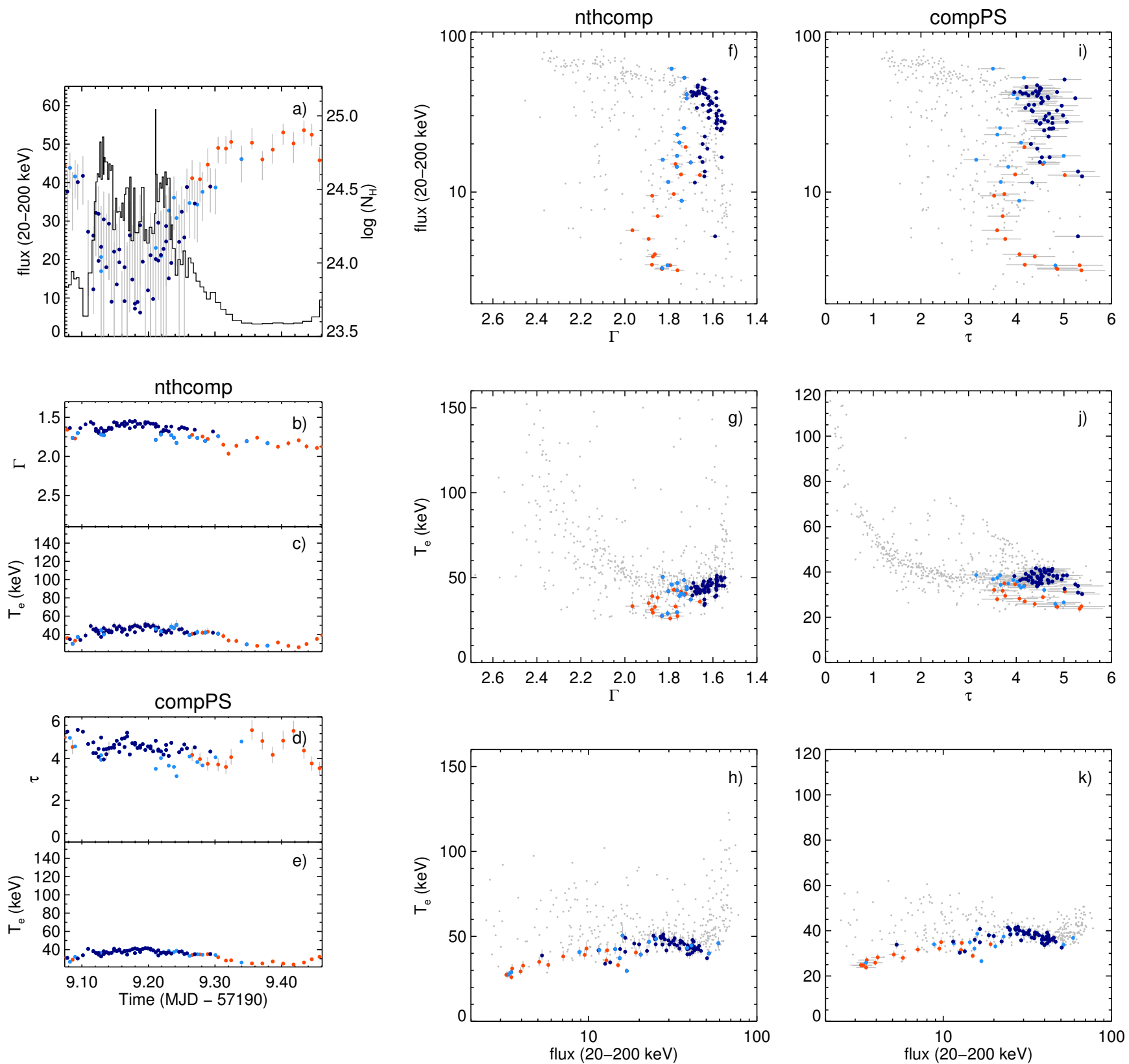

Fig. A.2. Close view of the properties of the hard X-ray flare and subsequent X-ray plateau detected around MJD 55199 , derived fitting the source spectra with Comptonization models (NTHCOMP and COMPPS). Panel a) time evolution of the source flux, $F_{\mathrm{x}}$, in the 20-200 keV energy range $\left(\times 10^{-8} \mathrm{erg} \mathrm{cm}^{-2} \mathrm{~s}^{-1}\right)$ in comparison with the $N_{\mathrm{H}}$ evolution, where $N_{\mathrm{H}}$ is derived by leaving it as a free parameter in our fits, as described in Sect. 3.4. During the peak of the flare, when $N_{\mathrm{H}}$ displays lower values, it cannot be constrained by our fits. Panels b)-e) time evolution of the spectral parameters derived for this period. Panels f)-h) relations between the various NTHCOMP spectral parameters during this period. Panels i)k) relations between the various COMPPS spectral parameters during this period. For reference, we also show in panels f)-k) the complete dataset analysed in this work (grey points). In the diagrams in panels f)-k) we observe parameter correlations characteristic of the hard branch (see sect. 3.3). The dramatic changes in flux observed during this X-ray flare are accompanied by little variations in $\Gamma$ and $\tau$. Brief transitions to the soft flaring branch are observed during the peak of the flare. During the flare decay, the spectrum gradually softens, $T_{\mathrm{e}}$ decreases and $\tau$ displays high variability, while we observe a gradual increase in $N_{\mathrm{H}}$, which reaches values close to $10^{25} \mathrm{~cm}^{-2}$ when the system reaches the bottom of the X-ray plateau. The spectral parameters derived during the X-ray plateau, occupy a separate region, as is evident in panels f)-h). Also, the parameter correlations reverse in the plateau branch with respect to the trends observed in the hard branch. 

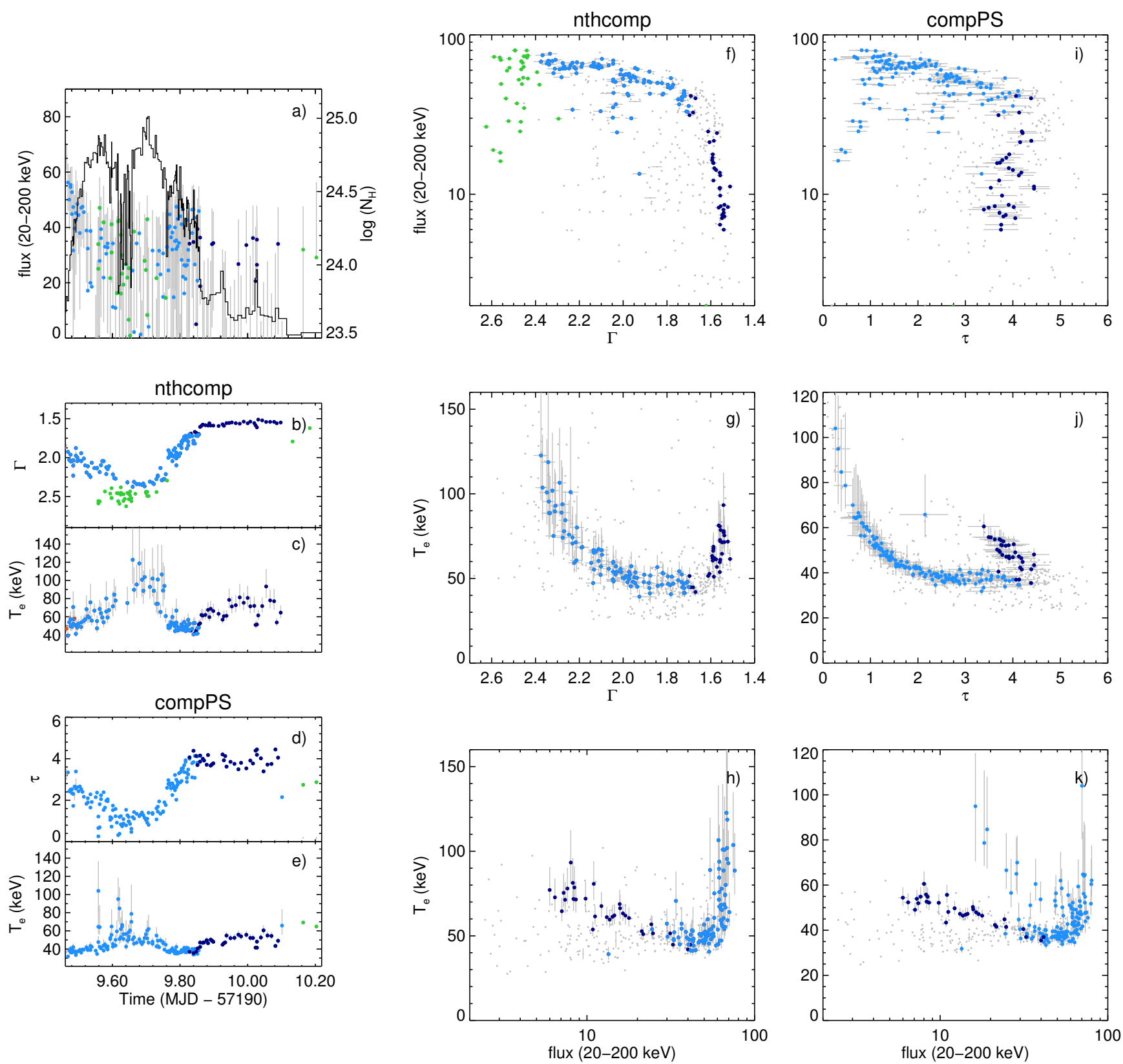

Fig. A.3. Close view of the properties of the soft X-ray flare detected around MJD 57 200, derived fitting the source spectra with Comptonization models (NTHCOMP and COMPPS). Panel a) time evolution of the source flux, $F_{\mathrm{x}}$, in the $20-200 \mathrm{keV}$ energy range $\left(\times 10^{-8} \mathrm{erg} \mathrm{cm}^{-2} \mathrm{~s}^{-1}\right)$ in comparison with the $N_{\mathrm{H}}$ evolution, where $N_{\mathrm{H}}$ was derived by leaving it as a free parameter in our fits, as described in Sect. 3.4. During the flare, when $N_{\mathrm{H}}$ displayed lower values, it could not be constrained by our fits. Panels b)-e) time evolution of the spectral parameters derived for this period. Panels f)-h) relations between the various NTHCOMP spectral parameters during this period. Panels i)-k) relations between the various COMPPS spectral parameters during this period. For reference, we also show in panels f)-k) the complete dataset analysed in this work (grey points). During the flare rise and decay we observed parameter correlations characteristic of the soft flaring branch (see panels f)-k), Sect. 3.3). Around the peak of the flare, the spectra softened above $\Gamma \sim 2.4$ and $T_{\mathrm{e}}$ was constrained using NTHCOMP. Spectral fits to these data using COMPPS provided un-constrained $T_{\mathrm{e}}$ values $\left(T_{\mathrm{e}} \gtrsim 100 \mathrm{keV}\right)$, and the lowest $\tau$ values found in this work $(\tau \lesssim 1)$. These spectra occupy a region in the $\Gamma-F_{\mathrm{x}}$ diagram (panel f)) reminiscent of the ultra-luminous state in the BHB HID. These spectra also occupy a separate branch in the COMPPS $F_{\mathrm{x}}-T_{\mathrm{e}}$ diagram (panel i)). After the peak of the flare, as $F_{\mathrm{x}}$ decreased and the spectrum hardened below $\Gamma \sim 2.4$ the system returned to the soft flaring branch. Around MJD 57199.85 a dramatic drop in flux was observed (from $F_{\mathrm{x}} \sim 50$ to $F_{\mathrm{x}} \sim 15 \times 10^{-8} \mathrm{erg} \mathrm{cm}^{-2} \mathrm{~s}^{-1}$ in about half an hour) and the system entered the hard branch. After this transition, the system started the decay to quiescence, following the hard branch characteristic correlations, but for a decreasing flux. 